\title{
Relationship between Urban Floristic Diversity and Ecosystem Services in the Moukonzi-Ngouaka Neighbourhood in Brazzaville, Congo
}

\author{
Victor Kimpouni ${ }^{1,2^{*}}(\mathbb{D}$, Josérald Chaîph Mamboueni², Ghislain Bileri-Bakala², \\ Charmes Maïdet Massamba-Makanda², Guy Médard Koussibila-Dibansa', Denis Makaya1 \\ ${ }^{1}$ École Normale Supérieure, Université Marien Ngouabi, Brazzaville, Congo \\ ${ }^{2}$ Institut National de Recherche Forestière, Brazzaville, Congo \\ Email: *vkimpouni@yahoo.com
}

How to cite this paper: Kimpouni, V., Mamboueni, J.C., Bileri-Bakala, G., Massamba-Makanda, C.M., Koussibila-Dibansa, G.M. and Makaya, D. (2020) Relationship between Urban Floristic Diversity and Ecosystem Services in the Moukonzi-Ngouaka Neighbourhood in Brazzaville, Congo. Open Journal of Ecology, 10, 788-821.

https://doi.org/10.4236/oje.2020.1012049

Received: September 16, 2020

Accepted: December 7, 2020

Published: December 10, 2020

Copyright $\odot 2020$ by author(s) and Scientific Research Publishing Inc. This work is licensed under the Creative Commons Attribution International License (CC BY 4.0).

http://creativecommons.org/licenses/by/4.0/ (c) (i) Open Access

\begin{abstract}
The relationship between ecosystem services and urban phytodiversity is being studied in the Moukonzi-Ngouaka district of Brazzaville. Urban forestry, a source of well-being for the inhabitants, is associated with socio-cultural foundations. The surveys concern flora, ethnobotany, socio-economics and personal interviews. The $60.30 \%$ naturalized flora is heterogeneous and closely correlated with traditional knowledge. The Guineo-Congolese endemic element groups are $39.27 \%$ of the taxa, of which $3.27 \%$ are native to Brazzaville. Ethnobotany recognizes $48.36 \%$ ornamental taxa; $28.36 \%$ food taxa; and $35.27 \%$ medicinal taxa. Some multiple-use plants are involved in more than one field. The supply service, a food and phytotherapeutic source, provides the vegetative and generative organs. Of the $52.31 \%$ of recognized taxa, $33.45 \%$ essentially provide leaves and $18.50 \%$ fruits. The main obsession of plant introduction being 1) food and phytotherapeutic, and 2) aesthetic, obscures other ecosystem services such as fuelwood and income, provided by NTFPs, pillars of poverty alleviation.
\end{abstract}

\section{Keywords}

Congo, Arboriculture, Urban Forestry, Traditional Knowledge,

Ecosystem Services, Floristic Diversity

\section{Introduction}

Throughout history, man has always shaped his habitat and/or the environment 
in his own image, consciously or unconsciously bringing exotic floral and wildlife elements [1]-[15]. These contributions, which can occur at any scale, profoundly and sometimes irreversibly modify the natural ecosystem [16] [17]. This quest for well-being is to the detriment of the natural biotope and biocenosis. This operation is at the origin of non-resilient systems in urban forestry, based on a monospecific or very heterogeneous floristic composition often marked by species represented by an individual.

The migration of plants is associated with the problematic of human movements, as several authors from the colonial and post-colonial period testify [1]-[15]. This observation is more than plausible, considering that Raponda-Walker and Sillans [2] report that Klaine, French missionary in Gabon, maintained in 1887 about 30 exotic tropical fruit trees (twelve varieties of grafted mangoes, Mangifera indica), cloves [Syzygium aromaticum (L.) Merr. \& Perry], vanilla (Vanilla planifolia Jackson), muscadier (Myristica fragrans Houtt.), about thirty ornamental trees and/or shrubs, ten varieties of rose bushes, etc.

The building of urban cities affects biodiversity in all its dimensions $(\alpha, \beta, \gamma)$ and indeed the related ecosystem services. The city of Brazzaville, founded in 1880, is no exception, since its original plant cover has disappeared and its floristic composition has been radically modified in quantity and quality [7] [18] [19] [20]. The combination of population explosion, amplified by rural exodus, and uncontrolled urbanization is the main cause. The enrichment of Brazzaville's flora with exotic species dates back to the 1880 s, as revealed by the maps kept at the Centre de recherche géographique et de production cartographique (CERGEC). They show Psidium guayava and Ananas sativus, two Neotropical non-native species introduced into Brazzaville as avenue plants.

The original plant formations that have now disappeared or are in very serious decline are of forest and savannah types. Until the 1970s, Brazzaville's natural vegetation consisted of five large independent forest areas (Figure 1), four of which (the Tsieme, the Glacial, the Corniche and the Chad Ravine) have disappeared without a trace of their biodiversity [21]. The Patte d'Oie forest, classified by Order No. 3037 of 13 August 1938 of the Governor General of French Equatorial Africa (AEF), placed about 240 ha of natural peri-urban forest in reserve. In spite of the reserve status and its biodiversity of scientific and cultural interest, this ecosystem has been strongly shaken by anthropisation. The decree $n^{\circ} 2009-149$ of 08 May 2009 in force reduces the area of this reserve to about 95 ha, integrating natural and artificial forests, administrative and university buildings, and sports facilities [22] [23] [24]. As a result, the natural forest cover of Brazzaville is estimated at less than $40 \%$ of its original area [22] [23] [24]. In addition to the patches resulting from Patte d'Oie forest degradation, the forest cover includes avenue trees, forming quickset hedges (Acacia mangium, Senna siamea, Terminalia superba, Terminalia mantaly, Peltophorum pterocarpum, Mangifera indica), monospecific plantations of Eucalyptus sp. and Pinus sp., and local species of mixed dense humid forests. Taxa counted in the latter system are 


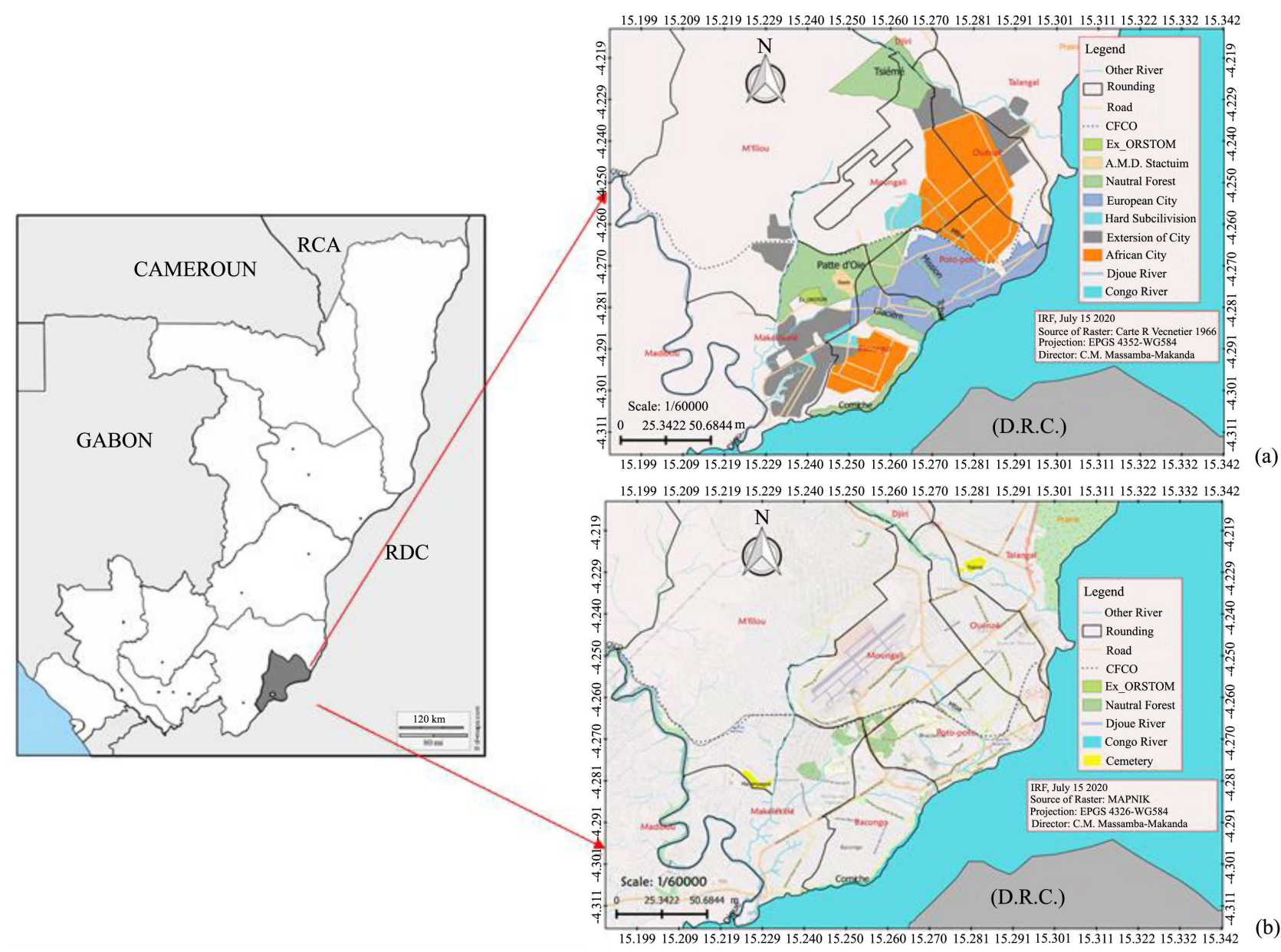

Figure 1. Scenes showing the regression of urban forest cover in Brazzaville over 50 years. Legend: source Vennetier, 1966 (a); source MAPNIK, 2016 (b).

Aucoumea klaineana, Milicia excelsa, Entandrophragma candollei, Entandrophragma cylindricum, Xylopia aethiopica, Alfzelia bipindensis, Syzygium brazzavillense, Syzygium guineensis, Millettia laurentii, Pterocarpus soyauxii, Lophira alata [14] [15] [25] [26]. As for the savannah flora, it has almost disappeared under the influence of urbanization, a corollary of the population growth that gave rise to the new districts. The herbaceous flora, typical of the savannah, is limited to a few rare wastelands and the presence of ruderal or wetland species.

The introduction of plants in Brazzaville is concomitant of interest to species not native to the Guineo-Congolese region (Aleurites moluccana, Bauhinia sp., Delonix regia, Senna siamea, Senna spectabilis, Lagerstroemia speciosa, Schizolobium parahybum, Jacaranda mimosoïdes, Hymenaea courbaril and Enterolobium cyclocarpum) and local species not native to the Léfini phytogeographical district such as Bridelia ferruginea, Canarium schweinfurthii, Sarcocephalus latifolius, Gardenia jovis-tonantis [3]. After the 1960s, this operation continued effectively with local taxa by indigenous populations. This craze is sustained primarily to satisfy the needs of traditional medicine and socio-cultural rituals. The corollaries of these contributions result in the disappearance of the original 
flora in favor of a flora with an exotic component, which is synonymous with anthropisation.

Urban cities in essence symbolize social success and therefore attract all social strata, even the poorest, to the quest for well-being [14] [15] [18] [19] [20]. In their study of alien flora and urban forestry in Brazzaville, Kimpouni et al. [14] [15] provide evidence of the change in floristic composition in a comprehensive manner and point out that this change would have a socio-cultural reason [27] [28]. Since the populations of residential and working-class neighborhoods do not have the same concerns, the introduction and adoption of plants does not have the same value and importance. Indeed, the interest and reason for the choice of taxa would be associated with the socio-cultural situation, even if there is a minor influence from the middle class and/or above [14] [15].

In view of the city's floristic development and the lack of basic data, given that the Congo does not have a long tradition of floristic inventories, undertaking a study of Brazzaville's indigenous and/or alien flora is a perilous exercise. Despite these shortcomings, this study is made possible by cross-referencing current urban forestry data with targeted surveys of managers and the city's population [29]. Current data focus on exotic taxa, to the point where the collective memory integrates them into the local flora without restraint. Thus, the local contributions associated with internal migration, which are less monitored, are clearly obscured. Within this body of knowledge, what is the footprint of local populations in the floristic change underway and for what benefits? The value and appreciation of this contribution is only possible by following the flora of the districts of the city of Brazzaville, and in this case, Moukonzi-Ngouaka is highly indicated. The introduction of exotic plants in Moukonzi-Ngouaka, one of the very old working-class neighbourhoods, is a hallmark of urban arboriculture in Congo. This study, which is part of the issue of man and his environment, provides information on the socio-cultural evidence underlying this phenomenon. Notwithstanding the interest aroused by urban forestry, the fact remains that the non-native floristic diversity, which is not subject to adequate chronological and taxonomic monitoring, is a worrying issue. This contribution aims to understand how the value of ecosystem services influences the enrichment of urban flora in Brazzaville, particularly in working-class neighborhoods. Its foundations are 1) the inventory of the flora of Moukonzi-Ngouaka, 2) the identification of the native region of the taxa and their current phytogeographic distribution, 3) the analysis of the services rendered to the community.

\section{Materials and Methods}

\subsection{Study Environment}

Brazzaville, located between $4^{\circ}-5^{\circ} \mathrm{S}$ and $15^{\circ}-16^{\circ} \mathrm{E}$ (Figure 2), extends for $1 / 3$ on a plain, formerly marshy, and for $2 / 3$ on a plateau $20 \mathrm{~m}$ higher, which dominates the Congo River [30] [31]. The climate is of the low Congolese type [30]; corresponding to the AW4 type according to Köppen's classification [32]-[37]. 


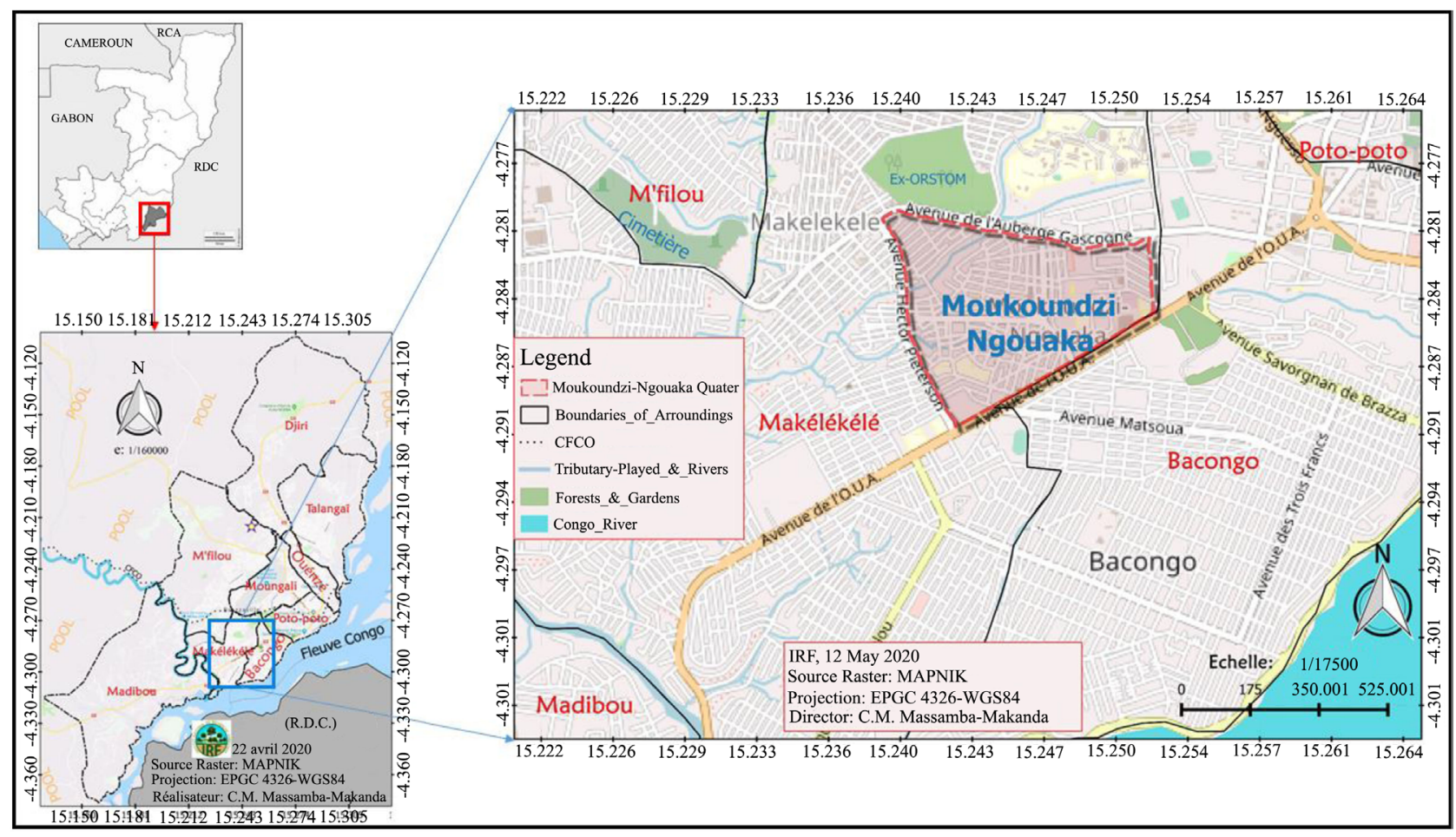

Figure 2. Location of the Moukonzi-Ngouaka district on the territory of Brazzaville.

Its characteristics can be summarized as: 1) a mean annual temperature close to $25^{\circ} \mathrm{C}$ (Figure 3 ) with a small thermal amplitude of $5^{\circ} \mathrm{C}$ to $6^{\circ} \mathrm{C}$. March and April are the hottest months, on the other hand, July and August are the coolest [38] [39]; 2) an average annual rainfall of $1200 \mathrm{~mm}$. Rainfall occurs from October to May with a very pronounced slowdown in January and February. The wettest months are generally March, April and November (Figure 3). The months of June to September are partly dry, resulting in stable air [31];3) a dry and cool season from June to September, which alternates with a wet and hot season from October to May [31]; 4) the relative humidity is particularly high, always above 70\%. An absolute minimum is noted in August and September, and a relative minimum in February and March. The minima vary between $50 \%$ and $60 \%$; the maxima vary between $88 \%$ and $94 \%$. Evaporation shows a relative maximum from February to March and an absolute maximum in August and September [21]; 5) the mean annual insolation oscillates between 1700 and 2000 hours. The insolation curve shows two maxima in April-May and November, and a minimum in July (Figure 3). Two tributaries of the Congo River, the Djiri in the north and the Djoué in the west water Brazzaville [31]. In addition to these main rivers, the Tsiémé in the north, Madoukou Tiékélé and the Mfoa in the centre flow into the Congo River. To the west, the M'filou is a tributary of the Djoué.

The vegetation consists of shrubby savanna and riparian forests rich in timber species. The work of Olson et al. [40] shows that the study site is located in the Afrotropic ecoregion and more precisely in the tropical and subtropical moist broadleaf forests zone. According to African chorology [41], this flora and 


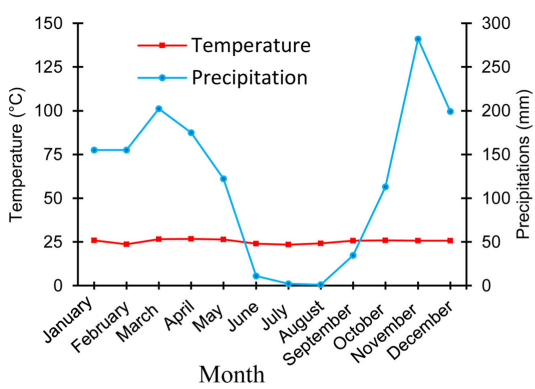

(a)

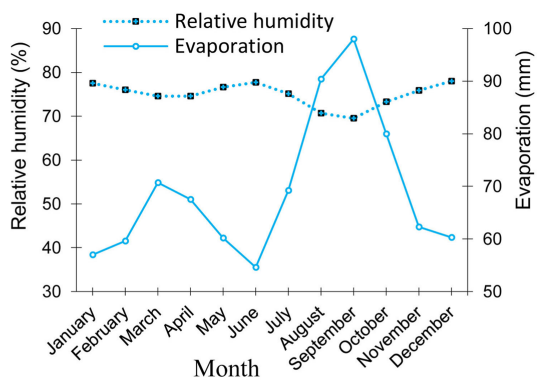

(b)

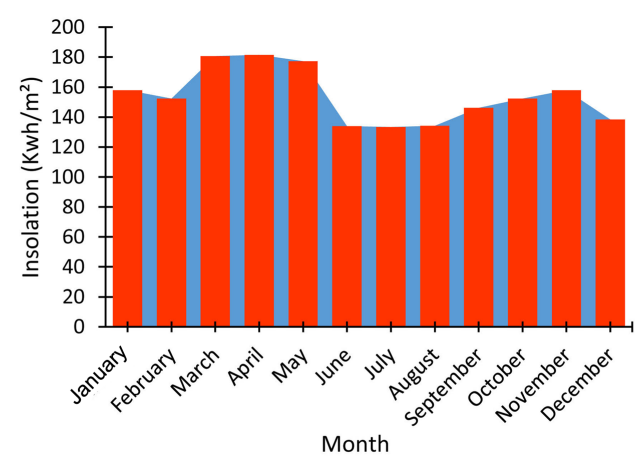

(c)

Figure 3. Climate parameters of the study area (2000-2018 period, source ANAC). Legend: Ombrothermal curve (a); Relative humidity and evaporation curves (b); Insolation (c).

vegetation belongs to the Guinean-Congolese Region, in particular to the Lower-Guinea endemism subcentre. By integrating Congo's phytogeographic data into the White [41] and Kimpouni et al. [42] show that the flora and vegetation of Brazzaville belongs to the Léfini District, in the Lower Congolo-Zambezian transition Sector of the Lower-Guinea Domain within the Guineo-Congolese Region. Thus, this vegetation, composed of shrubland, woodland and forests, corresponds to the drier peripheral semi-evergreen Guineo-Congolian rain forest, and Zambezian dry evergreen forest and transition woodland Grassland and wooded grassland [41]. Brazzaville's urban forest ecosystem is a low, m esophilic, tropophilic and dry formation. This ecosystem is almost without dominant strata, except for the one formed by introduced and generally isolated species such as Millettia laurentii, Albizia adianthifolia, Terminalia superba, Samanea saman, Hamanaea courbaril, Enterolobium cyclocarpum and Erythrophleum suaveolens. An atypical facies of African rainforest formations, the urban forest is made up of forest galleries and mesophilic islets resulting from the degradation of the Patte d'Oie forest [14] [15] [24]. In addition to these natural woody formations, Brazzaville has developed artificial forests, mainly of Eucalyptus sp. and Pinus sp. To this forest cover are added avenue trees (Acacia mangium, Terminalia superba, Terminalia mantaly, Peltophorum pterocarpum...).

The subsoil includes important series of mainly sandstone and sandy soils: the Batéké and Bambio plateau series (silicified sandstone, sands); the Stanley Pool series and the Carnot sandstones consisting of sandstone and argillite [31]. The 
soils of Brazzaville are ferrallitic, highly desaturated, impoverished and develop on sandy materials, poor in exchangeable bases, permeable to water [43].

\subsection{Study Material}

The plant material studied consists of woody and herbaceous species recorded in the Moukonzi-Ngouaka district. This floristic cohort is made up of non-native indigenous species of Brazzaville flora on the one hand, and allochtonous species from the Guineo-Congolese region on the other. The identification of the material is carried out in situ for common taxa and if necessary at the botany laboratory of the École normale supérieure (ENS) or even at the national herbarium (IEC), located at the Institut National de Recherche en Sciences Exactes et Naturelles (IRSEN) in Brazzaville. The basis for identification remains the floral fascicles of Gabon, Cameroon, Central Africa (DRC, Rwanda, Burundi) and Rwanda, Nzayilu Nti: Guide des arbres et arbustes de la région de Kinshasa-Brazzaville [44], the catalogue of vascular plants in Congo [45], Hortica [46] and Tropica [47], 1001 garden plants in Singapore [48]. At the taxonomic level: (1) the ordination of families follows the systems of APG IV [49] and PPG I [50]; (2) and the nomenclature adopted is that of Lebrun and Stork [51], for African taxa.

\subsection{Study Method}

It is based on two complementary phases, on the one hand, the bibliographical compilation and interviews with the owners and/or occupants of the parcels, and on the other hand the botanical prospection, parcel by parcel.

\subsubsection{Literature Review}

A systematic study on the state of knowledge of the original flora of Brazzaville, particularly the woody tree species, requires a good documentary research. Given the current level of urbanization, the practice of urban arboriculture is an activity that has profoundly changed the floristic landscape and flora [3] [6] [7] [14] [21] [22] [24] [26] [29] [52] [53]. The bibliographical analysis made it possible to list the species' origins and their current distribution, to follow the migratory trajectories, periods and history of introductions. Finally, it led to the detection of non-native species of the original flora of Brazzaville within the floristic procession.

The species inventoried are arranged into eight types of distribution, following the African chorological subdivisions [54]. These are Cosmopolitan (Cosm), Pantropical (Pant), Afro Malagasy (Afma), Afro tropical (Aftr), Afro American (Afam), Guinean-Congolese (GC), Lower-Guinean (CG), Palaeotropical (Paleo). As for the origin of exotic species at the centre of Guinean-Congolese endemism, the Holarctic, Neotropic, Paleotropic, Capensis and Australis empires are the main providers.

\subsubsection{Botanical Survey}

The botanical survey, which covers 3452 private properties (residential parcels 
with an average area of $400 \mathrm{~m}^{2}$, i.e. $20 \mathrm{~m} \times 20 \mathrm{~m}$ each), draws up an inventory of the plants counted. This inventory, which takes into account only private properties hosting plants, covers 1) the quickset hedges limiting these concessions and 2) of the courtyards of the dwellings in the said popular quarter. In the course of this inventory, the following information is provided for each species: status (naturalized, cultivated or spontaneous), number of individuals and biological forms [55] [56] [57]. The biological forms adopted to follow the classification of [58] and modified by Lebrun [59], for tropical regions. Their interest lies in determining adaptive strategies and vegetation physiognomy. The biological forms identified are mesophanerophyte (Msph), microphanerophyte (Mcph), nanophanerophyte (Nnph), climbing phanerophyte (Phgr), erect therophyte (Thd), climbing therophyte (Thr), climbing therophyte (Thgr), rhizomatous geophyte $(\mathrm{Gr})$, tuberous geophyte $(\mathrm{Gt})$, climbing tuberous geophyte (Gtgr), bulbous geophyte (Gb), erect chamephyte (Chd), prostrate chamephyte (Chr), hemicryptophyte (Hc).

\subsubsection{Surveys and Interviews}

The surveys are based on personal interviews with the owners and/or occupants of the parcels where the taxon is located. The interviews are semi-direct and open-ended, without influence on the expected response of the informant [60]. During this exercise, the approach consists, on the one hand, of identifying the resource and knowing its origin and associated properties, and, on the other hand, of acquiring data on the interest of introducing the taxon into Brazzaville. As in all research on endogenous knowledge, informants are very reluctant to share their knowledge, using the pretext that it will be developed without any value [61]. This is how 367 seniors responded to our request, without distinction of gender. Regardless of the gender, the informant had to have empirical knowledge about the benefits generated by the plants in his or her parcel or nearby. Thus, the selected informants were in the age range of at least 25 years and over.

\section{Results}

\subsection{Plant Data}

The floristic inventory (Table 1 and Figure 4) shows 281 species classified in three main groups: ferns (0.71\%); Gymnosperms (1.78\%); Magnoliids: Monocots (22.06\%), and Eudicots (75.44\%). According to the biological forms, this flora is divided into phanerophytes (63.57\%), chamephytes $(6.07 \%)$, geophytes $(9.64 \%)$, therophytes (17.50\%), and hemicryptophytes (3.21\%). The tree portion is dominated by nanophanerophytes $(28.21 \%)$, which are ahead of microphanerophytes (17.86\%) and mesophanerophytes (3.18\%). The proportion of mesophanerophytes is 3 times higher than the nanophanerophytes and microphanerophytes combined, in relation to the number of individuals counted. In terms of species diversity, the preponderant families are Fabaceae (10.32\%) and Euphorbiaceae (7.47\%). All other families (97.40\%) have a contribution between $0.36 \%$ and 
Table 1. General characteristics on the taxa inventoried.

\begin{tabular}{|c|c|c|c|c|c|c|c|}
\hline \multirow{2}{*}{$\mathrm{N}^{\circ}$} & Taxa & \multirow{2}{*}{ ТВ } & \multirow{2}{*}{ Statut } & \multirow{2}{*}{$\begin{array}{c}\text { Geographical } \\
\text { origin }\end{array}$} & \multirow{2}{*}{$\mathrm{TP}$} & \multirow{2}{*}{ Usage } & \multirow{2}{*}{$\begin{array}{c}\text { Ethnobotanic } \\
\text { Part use }\end{array}$} \\
\hline & Ferns & & & & & & \\
\hline & Nephrolepidaceae (2) & & & & & & \\
\hline 1 & Nephrolepis biserrata (Sw.) Schott & Hc. & spont. cult. & Tropical & Paleo & orn & - \\
\hline \multirow[t]{3}{*}{2} & Nephrolepis undulata (Afzel. ex Sw.) J. Sm. & Hc.. & spont. cult & Tropical & Pant & orn & - \\
\hline & Gymnosperms & & & & & & \\
\hline & Araucariaceae (2) & & & & & & \\
\hline 3 & Araucaria excelsa R. Br. & Msph. & cult. & Australia & Pant & orn. med. & $\mathrm{fe}$ \\
\hline \multirow[t]{2}{*}{4} & Araucaria sp. & Msph. & cult. & Australia & Pant & orn. & - \\
\hline & Cupressaceae (1) & & & & & & \\
\hline \multirow[t]{2}{*}{5} & Cupressus Iusitanica Mill. & Mcph. & cult & America & Cosm & orn & - \\
\hline & Cycadaceae (1) & & & & & & \\
\hline \multirow[t]{2}{*}{6} & Cycas revoluta Thunb. & Nnph. & cult. & Asia & Pant & orn & - \\
\hline & Pinaceae (1) & & & & & & \\
\hline \multirow[t]{4}{*}{7} & Pinus caribaea Morelet & Msph. & cult. & America & Pant & orn & - \\
\hline & Magnoliids & & & & & & \\
\hline & Monocots & & & & & & \\
\hline & Amaryllidaceae (1) & & & & & & \\
\hline \multirow[t]{2}{*}{8} & Amaryllis sp. & Gb. & cult. & - & - & orn & - \\
\hline & Araceae (11) & & & & & & \\
\hline 9 & Aglaonema commutatum Schott & Gr. & cult. & Asia & Pant & orn. & - \\
\hline 10 & Alocasia macrorrhiza (L.) G. Don & Gr. & cult. & Asia & Pant & orn. & - \\
\hline 11 & Caladium bicolor (Aiton) Vent. & Gt. & cult. & America & Pant. & orn. & - \\
\hline 12 & Caladium sp. & Gt. & cult. & America & Pant & orn. & - \\
\hline 13 & Colocasia esculenta (L.) Schott & Gr. & cult. & Asia & Pant. & alim. & Rh. fe \\
\hline 14 & Dieffenbachia amoena hort. ex Gentil & Chd. & cult & America & Pant & orn. & - \\
\hline 15 & Dieffenbachia regina W.Bull ex T. Moore \& Mast. & Chd. & cult. & America & Pant & orn. & - \\
\hline 16 & Epipremnum pinnatum (L.) Engl. & Phgr. & cult. & Asia & Pant & orn. & - \\
\hline 17 & Monstera deliciosa Liebm. & Phgr. & cult. & America & Pant & orn. & - \\
\hline 18 & Scindapsus aureus (Linden \& André) Engl. & Phgr. & cult. & Asia & Pant & orn. & - \\
\hline \multirow[t]{2}{*}{19} & Syngonium podophyllum $\mathrm{H}$. W. Schott & Phgr. & cult. & America & Pant & orn. & - \\
\hline & Arecaceae (4) & & & & & & \\
\hline 20 & Cocos nucifera $\mathrm{L}$. & Msph. & cult. & Asia & Pant. & alim. & Fr. \\
\hline 21 & Elaeis guineensis Jacq. & Msph. & cult. & Africa & Pant. & alim. & Fr. \\
\hline 22 & Phoenix reclinata Jacq. & Msph. & cult. & Africa & CG & alim. & Fr. \\
\hline 23 & Indet. & Msph. & cult. & - & - & orn. & - \\
\hline & Asparagaceae (6) & & & & & & \\
\hline
\end{tabular}




\section{Continued}

\begin{tabular}{|c|c|c|c|c|c|c|c|}
\hline 24 & Agave sisalana Perrine & Nnph. & cult. & America & Pant. & orn & - \\
\hline 25 & Asparagus angolensis Bak. & Nnph. & spont. cult. & Africa & GC & orn & - \\
\hline 26 & Cordyline fruticosa (L.) Chevalier & Nnph. & cult. & Asia & Pant & orn & - \\
\hline 27 & Dracaena fragrans (L.) Ker Gawl. & Nnph. & cult. & Africa & CG & orn & - \\
\hline 28 & Dracaena humilis Baker & Nnph. & cult. & Africa & CG & orn & - \\
\hline \multirow[t]{2}{*}{29} & Sansevieria trifasciata Prain & Chr. & cult. & Africa/Asia & Pant. & orn & - \\
\hline & Bromeliaceae (1) & & & & & & \\
\hline \multirow[t]{2}{*}{30} & Ananas sativus Schult \& Schult.f. & Chd. & cult. & America & Pant. & med. alim & Fr. \\
\hline & Cannaceae (1) & & & & & & \\
\hline \multirow[t]{2}{*}{31} & Canna indica $\mathrm{L}$. & Chr. & cult. & Asia/America & Pant & orn & - \\
\hline & Commelinaceae (3) & & & & & & \\
\hline 32 & Tradescantia spathacea Sw. & Chd. & cult. & America & pant & orn & - \\
\hline 33 & Tradescantia pallida (Rose) D. R. Hunt & Chd. & cult. & America & Pant. & orn & - \\
\hline \multirow[t]{2}{*}{34} & Tradescantia zebrina Bosse & Chr. & cult. & America & Pant. & orn & - \\
\hline & Costaceae (1) & & & & & & \\
\hline \multirow[t]{2}{*}{35} & Costus afer Ker-Gawl. & Gr. & spont. cult. & Africa & GC & med. & $\mathrm{t}$ \\
\hline & Cyperaceae (3) & & & & & & \\
\hline 36 & Cyperus articulatus $\mathrm{L}$. & Gr. & cult. & Africa & GC & med. & $\mathrm{Rh}$. \\
\hline 37 & Cyperus alternifolius $\mathrm{L}$. & Gr. & spont. cult. & Madagascar & Pant. & orn & - \\
\hline \multirow[t]{2}{*}{38} & Cyperus sp. & Gr. & cult. & - & - & orn & - \\
\hline & Dioscoreaceae (4) & & & & & & \\
\hline 39 & Dioscorea alata L. & Gtgr. & cult. & Asia & GC & alim. & Tub. \\
\hline 40 & Dioscorea bulbifera L. & Gtgr. & spont. cult. & Africa & Pant. & alim. & Tub. \\
\hline 41 & Dioscorea cayenensis Lamk. & Gtgr. & cult. & Africa & pant & alim. & Tub. \\
\hline \multirow[t]{2}{*}{42} & Dioscorea dumetorum (Kunth) Pax & Gtgr. & cult. & Africa & GC & alim. & Tub. \\
\hline & Heliconiaceae (2) & & & & & & \\
\hline 43 & Heliconia marginata (Griggs) Pittier & Gr. & cult. & America & Pant. & orn & - \\
\hline \multirow[t]{2}{*}{44} & Heliconia psittacorum L.f. & Gr. & cult. & America & Pant. & orn & - \\
\hline & Liliaceae (2) & & & & & & \\
\hline 45 & Allium schoenoprasum L. & Gb. & cult. & Europa/Asia & Cosm. & alim. & Bulbe. fe \\
\hline \multirow[t]{2}{*}{46} & Chlorophytum sp. & Gb. & spont. cult. & - & - & orn & - \\
\hline & Marantaceae (3) & & & & & & \\
\hline 47 & Hypselodelphys sp. & Phgr. & spont. cult. & Africa & GC & orn & - \\
\hline 48 & Maranta arundinacea L. & Gr. & cult. & America & & orn & - \\
\hline \multirow[t]{2}{*}{49} & Marantochloa mannii (Benth.) Milne-Redh. & Gr. & spont. cult. & Africa & GC & orn & - \\
\hline & Musaceae (2) & & & & & & \\
\hline 50 & Musa $x$ paradisiaca L. & Gr. & cult. & Asia & Pant & alim. & $\mathrm{fr}$ \\
\hline
\end{tabular}




\section{Continued}

\begin{tabular}{|c|c|c|c|c|c|c|c|}
\hline 51 & $\begin{array}{c}\text { Musa sapientum L. } \\
\text { Pandanaceae (1) }\end{array}$ & Gr. & cult. & Asia & Pant. & alim. med. & Fr. fe \\
\hline 52 & $\begin{array}{c}\text { Pandanus candelabrum P. Beauv. } \\
\text { Poaceae (11) }\end{array}$ & Mcph. & cult. & Africa & Pant. & orn. & - \\
\hline 53 & Anthephora cristata (Doell.) Hack. ex De Wild. & Thd. & spont. & Africa & GC & - & - \\
\hline 54 & Cymbopogon citratus (DC.) Stapf & Hc. & cult. & Asia & Pant. & alim. med. & Rh. fe \\
\hline 55 & Cymbopogon densiflorus (Steud.) Stapf & Hc. & cult. & Asia & GC & med. & $\mathrm{fe}$ \\
\hline 56 & Eleusine indica (L.) Gaertn. & Thd. & spont. & Asia & Pant. & med. & - \\
\hline 57 & Eragrostis tremula Hoscht. ex Steud. & Thd. & spont. & Africa/Asia & Cosm & - & - \\
\hline 58 & Panicum maximum Jacq. & Hc. & spont. & Asia & Afmal & - & - \\
\hline 59 & Paspalum notatum Fluegge & Hc. & cult. & America & Pant. & orn & - \\
\hline 60 & Saccharum officinarum $\mathrm{L}$. & Nnph. & cult. & Asia & Pant. & alim. & $\mathrm{t}$ \\
\hline 61 & Setaria megaphylla (Steud.) Dur. \& Schinz & Hc. & spont. cult. & Africa & Pant. & med. & $\mathrm{fe}$ \\
\hline 62 & Vetiveria zizanioides (L.) Nash & Hc. & spont. cult. & Asia & Pant. & orn & - \\
\hline 63 & $\begin{array}{c}\text { Zea mays } \mathrm{L} . \\
\text { Strelitziaceae }(1)\end{array}$ & Thd. & cult. & America & Cosm. & alim. med. & Stig. fe \\
\hline 64 & $\begin{array}{c}\text { Ravenala madagascariensis J.F. Gmel. } \\
\text { Zingiberaceae (5) }\end{array}$ & Mcph. & cult. & Madagascar & Pant. & orn & - \\
\hline 65 & Alpinia purpurata (Vieill.) K. Schum. & Nnph. & cult. & Oceania & Pant & orn & - \\
\hline 66 & Alpinia sanderae Hort. ex Sander & Nnph. & cult. & Oceania & Pant & orn & - \\
\hline 67 & Curcuma longa L. & Gr. & cult. & Asia & Pant. & orn & \\
\hline 68 & Zingiber officinale Roscoe & Gr. & cult. & Asia & Pant. & alim. med. & $\mathrm{Rh}$. \\
\hline 69 & $\begin{array}{c}\text { Zingiber zerumbet (L.) Roscoe ex Sm. } \\
\text { Eudicots } \\
\text { Acanthaceae (7) }\end{array}$ & Gr. & cult. & Asia & Pant. & orn & - \\
\hline 70 & Acanthus montanus (Nees) T. Anderson & Nnph. & cult. & Africa & GC & orn & - \\
\hline 71 & Brillantaisia patula T. Anders. & Nnph. & cult. & Africa & GC & med. & fe \\
\hline 72 & Hemigraphis colorata (Blume) H. G. Hallier & Nnph. & cult. & Asia & Pant & orn & - \\
\hline 73 & Justicia secunda Vahl & Nnph. & cult. & America & Pant & orn & - \\
\hline 74 & Ondontonema strictum (Nees) Kuntze & Nnph. & cult. & America & Pant. & orn. & - \\
\hline 75 & Sanchezia nobilis Hook.f. & Nnph. & cult. & America & pant & orn & - \\
\hline 76 & $\begin{array}{c}\text { Thunbergia erecta (Benth.) T. Anderson } \\
\text { Achariaceae (1) }\end{array}$ & Phgr. & cult. & Africa & CG & orn & - \\
\hline 77 & $\begin{array}{c}\text { Caloncoba welwitschii (Oliv.) Gilg } \\
\text { Adoxaceae (1) }\end{array}$ & Mcph. & cult. & Africa & CG & alim. med. & Fr. fe \\
\hline 78 & $\begin{array}{c}\text { Sambucus Mexicana C.Presl. ex DC. } \\
\text { Amaranthaceae (6) }\end{array}$ & Mcph. & cult. & America & Pant & med. orn & $\mathrm{fe}$ \\
\hline
\end{tabular}




\section{Continued}

\begin{tabular}{|c|c|c|c|c|c|c|c|}
\hline 79 & Alternanthera dentata (Moench) Stuchlik ex R. E. Fr. & Thr. & cult & America & Pant. & orn & - \\
\hline 80 & Amaranthus dibius Mart. ex Thell. & Thd. & spont/ cult. & Africa & AfAm & alim & fe \\
\hline 81 & Amaranthus hybridus $\mathrm{L}$. & Thd. & spont/ cult. & Asia & Afam & alim & fe \\
\hline 82 & Amaranthus viridis $\mathrm{L}$. & Thd. & spont. & Africa & Cosm. & alim & $\mathrm{fe}$ \\
\hline 83 & Amaranthus spinosus $\mathrm{L}$. & Thd. & spont. & Africa & Pant. & - & - \\
\hline \multirow[t]{2}{*}{84} & Celosia trigyna $\mathrm{L}$. & Thd. & spont. & Africa & pant & - & - \\
\hline & Anacardiaceae (4) & & & & & & \\
\hline 85 & Lannea welwitschii (Hiern) Engl. & Msph. & cult. & Africa & CG & med. & Éc.t. \\
\hline 86 & Mangifera indica $\mathrm{L}$. & Msph. & cult. & Asia & Pant. & alim. med. & Fr. Éc.t fe \\
\hline 87 & Spondias mombin L. & Msph. & cult. & America & Pant. & alim. med. & Fr. fe \\
\hline \multirow[t]{2}{*}{88} & Spondias dulcis Forst F. & Msph. & cult. & America & Paléo. & alim & Fr. \\
\hline & Annonaceae (3) & & & & & & \\
\hline 89 & Annona muricata L. & Mcph. & cult. & America & Pant. & alim. med. & Fr. Éc.t Fe \\
\hline 90 & Annona squamosa L. & Mcph. & cult. & America & Pant. & alim. & Fr. \\
\hline \multirow[t]{2}{*}{91} & Cananga odorata (Lam.) Hook.f. & Msph. & cult. & Asia & Pant. & orn & - \\
\hline & Apocynaceae (7) & & & & & & \\
\hline 92 & Allamanda cathartica L. & Nnph. & cult. & America & Pant. & orn & - \\
\hline 93 & Catharanthus roseus (L.) G.Don & Chd. & cult. & Madagascar & Pant. & orn & - \\
\hline 94 & Plumeria alba L. & Mcph. & cult. & America & Pant. & orn & - \\
\hline 95 & Plumera rubra L. & Mcph. & cult. & America & Pant. & orn & - \\
\hline 96 & Rauvolfia mannii Stapf & Nnph. & cult. & Africa & CG & med. orn & Ra. Fr. Éc.r. fe \\
\hline 97 & Saba comorensis (Bojer) Pichon & Phgr. & cult. & Africa & Aftr. & alim. & Fr. \\
\hline \multirow[t]{2}{*}{98} & Thevetia peruviana (Pers.) K. Schum. & Nnph. & cult. & America & Pant & orn. & - \\
\hline & Araliaceae (4) & & & & & & \\
\hline 99 & Polyscia balfouriana (André) L.H.Bailey & Nnph. & cult. & Polynesia & Pant & orn. & - \\
\hline 100 & Polyscia guilfoylei L. H. Bailey & Nnph. & cult. & Polynesia & Pant & orn & - \\
\hline 101 & Polyscias paniculata (DC.) Baker & Nnph. & cult. & Polynesia & Pant & orn. & - \\
\hline \multirow[t]{2}{*}{102} & Polyscias scutellaria (Burm. f.) Fosberg & Nnph. & cult. & Polynesia & Pant & orn. & - \\
\hline & Aristolochiaceae (1) & & & & & & \\
\hline \multirow[t]{2}{*}{103} & Aristolochia sp. & Phgr. & spont. & Africa & - & orn. & - \\
\hline & Asteraceae (14) & & & & & & \\
\hline 104 & Acanthospermum hispidium DC. & Thd. & spont. & America & Pant. & med. & $\mathrm{fe}$ \\
\hline 105 & Acmella caulirhiza Delile & Thr. & spont. & America & Pant. & med. & $\mathrm{fe}$ \\
\hline 106 & Ageratum conyzoides $\mathrm{L}$. & Thd.. & spont & America/Asia & Pant. & med. & $\mathrm{fe}$ \\
\hline 107 & Aspilia kotschyi (Sch.Bip.) Oliv. & Thd. & spont. & Africa & Pant. & orn & - \\
\hline 108 & Bidens pilosa $\mathrm{L}$. & Thd. & spont. & America & Pant. & med. & fe \\
\hline 109 & Chromolaena odorata (L.) King \& Robinson & Nnph. & spont. & America & Pant. & med. & $\mathrm{fe}$ \\
\hline
\end{tabular}




\section{Continued}

\begin{tabular}{|c|c|c|c|c|c|c|c|}
\hline 110 & Conyza sumatrensis (Retz) E. Walker. & Thd. & spont. & America & Pant. & med. & fe \\
\hline 111 & Emilia sonchifolia (L.) DC & Thd. & spont. & Asia & Pant. & med. & fe \\
\hline 112 & Gymnanthemum amygdalinum (Delile) Walp. & Mcph. & spont. cult. & Africa & Aftr. & med. orn. & fe \\
\hline 113 & Helianthus annuus $\mathrm{L}$. & Thd. & cult. & America & Cosm. & orn & - \\
\hline 114 & Solanecio angulatus (Vahl) C. Jeffrey & Thgr. & spont. & Africa & GC & alim. & fe \\
\hline 115 & Synedrella nodiflora (L.) Gaertn & Thd. & spont. & America & CG & med & - \\
\hline 116 & Tagetes sp. & Thd. & spont. & - & - & orn & - \\
\hline \multirow[t]{2}{*}{117} & Tithonia diversifolia (Hemsl.) Gray & Nnph. & spont. cult. & America & Pant. & med. orn. & $\mathrm{fe}$ \\
\hline & Balsaminaceae (1) & & & & & & \\
\hline \multirow[t]{2}{*}{118} & Impatiens irvingii Hook.f. ex Oliv. & Chd. & spont. cult. & Africa & Aftr. & orn & - \\
\hline & Basellaceae (2) & & & & & & \\
\hline \multirow[t]{2}{*}{119} & Basella rubra L. & Chgr. & cult. & Asia & Pant. & alim. & $\mathrm{fe}$ \\
\hline & Begoniaceae (2) & & & & & & \\
\hline 120 & Begonia sp. 1 & Chd. & cult. & - & - & orn & - \\
\hline \multirow[t]{2}{*}{121} & Begonia sp. 2 & Chd. & cult. & - & - & orn & - \\
\hline & Bignoniaceae (3) & & & & & & \\
\hline 122 & Markhamia tomentosa (Benth.) K. Schum. ex Engl. & Mcph. & spont. cult. & Africa & GC & med. orn & Éc.t. \\
\hline 123 & Newbouldia laevis (P.Beauv.) Seen ex Bureau & Mcph. & cult. & Africa & GC & med. orn. & Éc.r. ra. fe \\
\hline \multirow[t]{2}{*}{124} & Tabebuia rosea (Bertol.) DC & Msph. & cult. & America & Pant & orn & - \\
\hline & Bixaceae (1) & & & & & & \\
\hline \multirow[t]{2}{*}{125} & Bixa Orellana L. & Mcph. & cult. & America & Pant. & orn & - \\
\hline & Brassicaceae (2) & & & & & & \\
\hline 126 & Brassica sp. 1 & Thd. & cult. & Eurasia & Pant & alim & fe \\
\hline \multirow[t]{2}{*}{127} & Brassica sp. 2 & Thd. & cult. & Eurasia & Pant & alim & fe \\
\hline & Burseraceae (2) & & & & & & \\
\hline 128 & Dacryodes edulis (G. Don) Lam. & Msph. & cult. & Africa & CG & med. alim & Fr. Éc. fe \\
\hline \multirow[t]{2}{*}{129} & Canarium schweinfurthii Engl. & Msph. & cult. & Africa & GC & med. alim & Fr. \\
\hline & Cactaceae (2) & & & & & & \\
\hline 130 & Cereus peruvianus (L.) Miller & Mcph. & cult. & America & Pant & orn & - \\
\hline \multirow[t]{2}{*}{131} & Cereus peruvianus var. monstruosus DC. & Mcph. & cult. & America & Pant & orn & - \\
\hline & Cannabaceae (2) & & & & & & \\
\hline 132 & Cannabis sativa $\mathrm{L}$. & Nnph. & cult. & Asia & Pant. & med. & fe \\
\hline \multirow[t]{2}{*}{133} & Trema orientalis $(\mathrm{L}$.$) Blume$ & Mcph. & spont.cult. & Africa & Pant & med. & Ra. \\
\hline & Capparidaceae (2) & & & & & & \\
\hline 134 & Buchholzia macrophylla Pax & Mcph. & cult & Africa & CG & med & fr \\
\hline \multirow[t]{2}{*}{135} & Sieruela rutidosperma (DC.) Roalson \& J. C. Hall & Thd. & spont. & Africa & Paléo. & med. & $\mathrm{fe}$ \\
\hline & Caricaceae (1) & & & & & & \\
\hline
\end{tabular}




\section{Continued}

\begin{tabular}{|c|c|c|c|c|c|c|c|}
\hline 136 & $\begin{array}{c}\text { Carica papaya L. } \\
\text { Caryophyllaceae (1) }\end{array}$ & Mcph. & spont. cult. & America & Pant & alim. med. & Fr. Ra. Gr. fe \\
\hline 137 & $\begin{array}{c}\text { Drymaria cordata (L.) Willd. ex Schult. } \\
\text { Chenopodiaceae (1) }\end{array}$ & Thr. & spont. & America & Pant. & med & $\mathrm{fe}$ \\
\hline 138 & $\begin{array}{c}\text { Chenopodium ambrosioides } \mathrm{L} . \\
\text { Clusiaceae (1) }\end{array}$ & Thd. & spont. & America & Pant & med. & fe \\
\hline 139 & $\begin{array}{l}\text { Garcinia mannii Oliv. } \\
\text { Combretaceae (5) }\end{array}$ & Mcph. & cult. & Africa & GC & alim. & Fr. \\
\hline 140 & Combretum racemosum P. Beauv. & Phgr. & spont. cult. & Africa & GC & med. & $\mathrm{fe}$ \\
\hline 141 & Quisqualis indica $\mathrm{L}$. & Phgr. & spont. cult. & Asia & Pant & orn & - \\
\hline 142 & Terminalia catappa L. & Msph. & cult. & Asia & Pant. & alim. & Fr. \\
\hline 143 & Terminalia mentaly H. Perrier & Msph. & cult. & Asia & & orn & \\
\hline 144 & $\begin{array}{l}\text { Terminalia superba Engl. } \\
\text { Connaraceae (1) }\end{array}$ & Msph. & spont. cult. & Africa & GC & orn & - \\
\hline 145 & $\begin{array}{c}\text { Manotes pruinosa Gilg } \\
\text { Convolvulaceae (4) }\end{array}$ & Phgr. & spont. cult. & Africa & CG & med. & $\mathrm{fe}$ \\
\hline 146 & Ipomoea batatas (L.) Lam. & Phr. & cult. & America & Pant. & alim. & Tub. \\
\hline 147 & Ipomoea carnea Jacq. & Nnph. & cult. & America & Pant. & orn & - \\
\hline 148 & Ipomoea nil (L.) Roth & Thgr. & cult. & America & Pant. & orn & - \\
\hline 149 & $\begin{array}{c}\text { Ipomoea quamoclit } \mathrm{L} \text {. } \\
\text { Crassulaceae }(2)\end{array}$ & Thgr. & spont. cult. & America & Pant. & orn & - \\
\hline 150 & Bryophyllum pinnatum (Lam.) Oken & Nnph. & cult & Madagascar & Aftr. & med. & $\mathrm{fe}$ \\
\hline 151 & $\begin{array}{c}\text { Kalonchoe crenata (Andr.) Harv. } \\
\text { Cucurbitaceae (6) }\end{array}$ & Nnph. & cult. & Madagascar & Aftr. & med. & $\mathrm{fe}$ \\
\hline 152 & Citrullus sp. & Thr. & spont. & Africa & Cosm & alim. & $\mathrm{fe}$ \\
\hline 153 & Coccinia barteri (Hook.f.) Keay & Thgr. & spont. & Africa & CG & alim. & $\mathrm{fe}$ \\
\hline 154 & Coccinia grandis (L.) Voigt & Thgr. & spont. & Africa/Asia & CG & alim. & $\mathrm{fe}$ \\
\hline 155 & Cucurbita pepo L. & Thgr. & spont.cult. & America & Pant & alim. & $\mathrm{fe}$ \\
\hline 156 & Luffa aegyptiaca Mill. & Thgr. & spont. cult. & Asia & Pant & alim. med. & $\mathrm{fe}$ \\
\hline 157 & $\begin{array}{c}\text { Momordica charantia L. } \\
\text { Ebenaceae }(1)\end{array}$ & Thgr. & spont. & Africa & Pant. & alim. med. & Fr. fe \\
\hline 158 & $\begin{array}{c}\text { Diospyros heterotricha (B.L.Burtt) F. White } \\
\text { Euphorbiaceae (21) }\end{array}$ & Mcph. & cult. & Africa & GC & med. & Fr. Ra. éc \\
\hline 159 & Acalypha amentacea Roxb. & Nnph. & cult. & Australia & Pant & orn & - \\
\hline 160 & $\begin{array}{l}\text { Acalypha amentacea Roxb. subsp. } \\
\text { wilkesiana (Müll.Arg.) Fosberg }\end{array}$ & Nnph. & cult. & Australia & Pant. & orn & - \\
\hline 161 & Acalypha hispida Burm.f. & Nnph. & cult. & Australia & Pant. & orn & - \\
\hline 162 & Acalypha marginata J. J. Sm. & Nnph. & cult. & Australia & pant & orn & - \\
\hline
\end{tabular}


Continued

\begin{tabular}{|c|c|c|c|c|c|c|c|}
\hline 163 & Acalypha peduncularis E.Mey. ex Meisn. & Nnph. & cult. & Australia & Pant. & orn & - \\
\hline 164 & Alchornea cordifolia (Schumach. \& Thonn.) Muell. Arg. & Mcph. & spont. cult. & Africa & Aftr. & med. & fe \\
\hline 165 & Codiaeum variegatum (L.) Juss. & Nnph. & cult. & Asie & Pant & orn & - \\
\hline 166 & Croton sp. & Nnph. & cult. & Asie & Pant & orn & - \\
\hline 167 & Euphorbia candelabrum Kotschy & Mcph. & cult. & America & Pant & orn & - \\
\hline 168 & Euphorbia cotinifolia L. & Nnph. & cult. & America & Pant. & orn & - \\
\hline 169 & Euphorbia hirta L. & Thd. & cult. & Tropical ? & GC & med & - \\
\hline 170 & Euphorbia prostrata Ait & Thd. & cult. & America & GC & med & - \\
\hline 171 & Euphorbia pulcherrima Willd. ex Klotzsch & Nnph. & cult. & America & Pant. & orn & - \\
\hline 172 & Euphorbia thymifolia L. & Thd. & cult. & Africa & GC & med & - \\
\hline 173 & Euphorbia tirucalli $\mathrm{L}$. & Nnph. & cult. & Africa & paleo & orn & - \\
\hline 174 & Hura crepitans L. & Mcph. & cult. & America & Pant & med. & Gr. fe \\
\hline 175 & Jatropha curcas $\mathrm{L}$. & Nnph. & cult. & America & Pant & med. & fe \\
\hline 176 & Manihot esculenta Crantz & Nnph. & cult. & America & Pant. & alim. & Ra. fe \\
\hline 177 & Manihot glaziovii Muell.Arg. & Nnph. & cult. & America & Pant. & alim. & $\mathrm{fe}$ \\
\hline 178 & Pedilanthus tithymaloides $\mathrm{L}$. & Nnph. & cult. & America & Pant. & orn & - \\
\hline \multirow[t]{2}{*}{179} & Ricinus communis $\mathrm{L}$. & Nnph. & cult. & Asia & Pant & orn & - \\
\hline & Fabaceae (29) & & & & & & \\
\hline 180 & Abrus precatorius $\mathrm{L}$. & Thgr. & spont. cult. & Africa / Asia & Afmal & med. & $\mathrm{fe}$ \\
\hline 181 & Acacia leucocephala (Lam.) Link & Mcph. & spont. cult. & Asia & Pant & orn & - \\
\hline 182 & Acacia mangium Willd. & Mcph. & spont. cult. & Australia & Pant. & orn & - \\
\hline 183 & Albizia lebeck (L.) Benth. & Mcph. & cult. & Asia & Pant & orn & - \\
\hline 184 & Arachis hypogaea L. & Thd. & cult. & America & Pant. & alim. & Fr. \\
\hline 185 & Bauhinia tomentosa $\mathrm{L}$. & Nnph. & cult. & Asia & Pant & orn & - \\
\hline 186 & Caesalpinia pulcherrima (L.) Sw. & Nnph. & cult. & Asia & Pant & orn & - \\
\hline 187 & Cajanus cajan (L.) Millsp. & Nnph. & cult. & Africa & Pant. & alim. & Fr. \\
\hline 188 & Calliandra surinamensis Benth. & Nnph. & cult. & America & Pant. & orn & - \\
\hline 189 & Canavalia ensiformis (L.) DC. & Thgr. & cult. & America & Pant & med. & Fr. \\
\hline 190 & Cassia javanica L. & Mcph. & cult. & Asia & Pant. & orn. & - \\
\hline 191 & Delonix regia (Bojer) Rafin & Msph. & cult. & Madagascar & Pant. & orn & - \\
\hline 192 & Desmodium velutinum (Sw.) DC. & Nnph. & spont. & & paléo & med. & fe \\
\hline 193 & Dichrostachys glomerata (Forsk.) Chiov. & Mcph. & spont. cult. & Africa & Paléo. & med. & fe \\
\hline 194 & Erythrophleum suaveolens (Guill. \& Perr.) Brenan & Msph. & cult. & Africa & GC & med. & Ec.t. éc.ra. fe \\
\hline 195 & Millettia eetveldeana (Micheli) Hauman & Mcph. & spont. cult. & Africa & CG & med. & $\mathrm{fe}$ \\
\hline 196 & Millettia laurentii De Wild. & Msph. & spont. cult. & Africa & CG & med. & Éc.t fe \\
\hline 197 & Millettia versicolor Welw. ex Baker & Mcph. & spont. cult. & Africa & CG & med. & Ra. Ec.t \\
\hline 198 & Mimosa pudica L. & Nnph. & spont. & America & GC & med & - \\
\hline 199 & Mucuna sp. & Thgr. & spont. & - & & orn & \\
\hline
\end{tabular}




\section{Continued}

\begin{tabular}{|c|c|c|c|c|c|c|c|}
\hline 200 & Peltophorum pterocarpum (DC.) K. Hyene & Msph. & cult. & Asia & Pant. & med & Ec. \\
\hline 201 & Pentaclethra eetveldeana De Wild. ex Th. Dur. & Msph. & spont. cult. & africa & CG & med. & Ec.t \\
\hline 202 & Phaseolus vulgaris $\mathrm{L}$. & Thgr. & cult. & America & Pant. & alim. & fr \\
\hline 203 & Psophocarpus palustris Desv. & Thgr. & spont. cult. & Africa & GC & alim. & fe \\
\hline 204 & Senna alata (L.) Roxb & Nnph. & cult. & America & Pant. & med. & fe \\
\hline 205 & Senna occidentalis (L.) Link & Nnph. & spont. cult. & Africa & Pant. & med. & Fr. fe. Ra. \\
\hline 206 & Senna spectabilis (DC.) H. S. Irwin \& Barneby & Mcph. & cult. & America & pant & orn & - \\
\hline 207 & Senna tora (L.) Roxb & Nnph. & spont. & Asia & Pant. & med. & $\mathrm{fe}$ \\
\hline \multirow[t]{2}{*}{208} & Tephrosia vogelii Hook. f. & Nnph. & spont. cult. & Africa & Aftr. & med. & fe \\
\hline & Gentianaceae (1) & & & & & & \\
\hline \multirow[t]{2}{*}{209} & Anthocleista schweinfurthii Gilg & Msph. & spont. & Africa & Aftr. & med. & Fr. fe \\
\hline & Gesneriaceae (1) & & & & & & \\
\hline \multirow[t]{2}{*}{210} & Episcia cupreata (Hook.) Hanst. & Nnph. & cult. & America & Pant. & orn & - \\
\hline & Lamiaceae (6) & & & & & & \\
\hline 211 & Clerodendrum inerme (L.) Gaertn. & Phgr. & cult. & Asia & Pant & med. orn & - \\
\hline 212 & Clerodendrum splendens $\mathrm{G}$. Don & Phgr. & spont.cult & Africa & GC & orn & - \\
\hline 213 & Ocimum basilicum $\mathrm{L}$. & Nnph. & cult. & Asia & Pant. & alim. & fe \\
\hline 214 & Ocimum canum Sims & Nnph. & cult. & Africa/Asia & Pant. & med. & fe \\
\hline 215 & Ocimum gratissimum $\mathrm{L}$. & Nnph. & cult. & Asia & paléo & med. & fe \\
\hline \multirow[t]{2}{*}{216} & Coleus sp. & Chd. & cult. & - & - & orn & - \\
\hline & Lauraceae (1) & & & & & & \\
\hline \multirow[t]{2}{*}{217} & Persea americana Mill. & Msph. & cult. & America & Pant. & alim. med. & Fr. fe \\
\hline & Malvaceae (12) & & & & & & \\
\hline 218 & Abelmoschus esculentus (L.) Moench & Nnph. & cult. & Africa & Pant & alim. & Fr. \\
\hline 219 & Ceiba pentandra (L.) Gaertn. & Msph. & spont.cult & America & Pant. & med. & Ec.t. \\
\hline 220 & Cola acuminata (P.Beauv.) Schott \& Endl. & Mcph. & cult. & Africa & GC & alim. & fr \\
\hline 221 & Corchorus olitorius $\mathrm{L}$. & Thd. & spont.cult. & Asia & Pant. & alim. & fe \\
\hline 222 & Glyphaea brevis (Spreng.) Monachino & Nnph. & cult. & Africa & Aftr. & alim. & fe \\
\hline 223 & Gossypium barbadense L. & Nnph. & cult. & America & Pant. & med. & Fr. fe \\
\hline 224 & Hibiscus rosa sinensis $\mathrm{L}$. & Nnph. & cult. & Asia & Cosm. & orn & - \\
\hline 225 & Hibiscus sabdariffa L. & Nnph. & spont. cult. & Africa & Pant & alim. & Fr. fe \\
\hline 226 & Malvaviscus arboreus Dill. ex Cav. & Nnph. & cult. & America & Pant. & orn & - \\
\hline 227 & Pachira glabra Pasq. & Mcph. & cult. & Asia & Pant & alim & $\mathrm{fr}$ \\
\hline 228 & Theobroma cacao L. & Mcph. & cult. & America & Pant. & alim. & fr \\
\hline \multirow[t]{2}{*}{229} & Urena lobata L. & Nnph. & spont. & America & Pant. & med. & fe \\
\hline & Moraceae (6) & & & & & & \\
\hline 230 & Artocarpus altilis (Parkinson) Fosberg & Msph. & cult. & Asia & Pant. & alim. & fr \\
\hline 231 & Ficus benjamina L. & Mcph. & cult. & Asia & Pant & orn & - \\
\hline
\end{tabular}




\section{Continued}

\begin{tabular}{|c|c|c|c|c|c|c|c|}
\hline 232 & Ficus sur Forssk. & Mcph. & cult. & Africa & CG & med. & Éc.t fe \\
\hline 233 & Ficus elastica Roxb. & Mcph. & cult. & Asia & Pant. & orn & \\
\hline 234 & Ficus sp. & Mcph. & spont. cult. & - & - & med. & $\mathrm{fe}$ \\
\hline \multirow[t]{2}{*}{235} & Trilepisium madagascariense DC. & Mcph. & spont. cult. & Africa & Afmal & alim. med. & Fr. Éc.t \\
\hline & Myrtaceae (4) & & & & & & \\
\hline 236 & Eucalyptus sp. & Msph. & cult. & Australia & Pant. & orn & - \\
\hline 237 & Eucalyptus tereticornis J. E. Sm. & Msph. & cult. & Australia & Pant. & orn. med. & $\mathrm{fe}$ \\
\hline 238 & Eugenia uniflora L. & Mcph. & cult. & America & Pant. & alim. med. & Fr. fe \\
\hline \multirow[t]{2}{*}{239} & Psidium guajava $\mathrm{L}$. & Mcph. & spont. cult. & America & Pant. & alim. med. & Fr. Éc.t \\
\hline & Nyctagynaceae (3) & & & & & & \\
\hline 240 & Boerhavia diffusa $\mathrm{L}$. & Gt. & spont & Africa & Pant. & med. & $\mathrm{fe}$ \\
\hline 241 & Bougainvillea glabra Choisy & Phgr. & cult. & America & Cosm. & orn & - \\
\hline \multirow[t]{2}{*}{242} & Mirabilis jalapa L. & Thd. & spont. cult. & America & Pant. & orn & - \\
\hline & Passifloraceae (2) & & & & & & \\
\hline 243 & Passiflora edulis Sims. & Phgr. & cult. & America & Pant. & alim. & Fr. \\
\hline \multirow[t]{2}{*}{244} & Passiflora quadrangularis $\mathrm{L}$. & Phgr. & cult. & America & Pant. & alim. & Fr. \\
\hline & Periplocaceae (1) & & & & & & \\
\hline \multirow[t]{2}{*}{245} & Mondia whitei (Hook. f.) Skeels & Phgr. & cult. & Africa & GC & alim. med. & Ra. \\
\hline & Phyllanthaceae (3) & & & & & & \\
\hline 246 & Bridelia ferruginea Benth. & Nnph. & cult. & Africa & Aftr. & med. & Éc t. éc ra \\
\hline 247 & Hymenocardia acida Tul. & Nnph. & cult. & Africa & GC & med. & Éc $t$ \\
\hline \multirow[t]{2}{*}{248} & Phyllanthus articulatus Poir. & Nnph. & cult. & Africa & CG & med. orn & fe \\
\hline & Piperaceae (2) & & & & & & \\
\hline 249 & Piper guineense Schum. \& Thonn. & Phgr. & cult. & Africa & GC & alim. & $\mathrm{fe}$ \\
\hline \multirow[t]{2}{*}{250} & Peperomia molleri C.DC & Chd. & spont. cult. & Africa & Pant. & orn & - \\
\hline & Polygonaceae (2) & & & & & & \\
\hline 251 & Antigonum leptopus Hook. f. \& Arn. & Phgr. & cult. & America & Pant. & orn & - \\
\hline \multirow[t]{2}{*}{252} & Rumex abyssinicus Meisn. & Nnph. & spont. cult. & Africa & Pant & alim. & fe \\
\hline & Portulaccaceae (2) & & & & & & \\
\hline 253 & Portulaca oleracea L. & Thr. & spont. cult. & Asia? & Cosm. & med. orn & $\mathrm{fe}$ \\
\hline \multirow[t]{2}{*}{254} & Talinum triangulare (Jacq.) Willd. & Thd. & spont. & America? & Pant. & alim. & $\mathrm{fe}$ \\
\hline & Rubiaceae (5) & & & & & & \\
\hline 255 & Gardenia jovis-tonantis (Welw.) Hiern & Nnph. & cult. & Africa & Aftr. & med. & Ra. $t$ \\
\hline 256 & Heinsia crinita (Afz.) G. Tayl. & Nnph. & spont. cult. & Africa & Aftr. & med. & Ra. fe \\
\hline 257 & Ixora coccinea $\mathrm{L}$. & Nnph. & cult. & Asia & Pant & orn & - \\
\hline 258 & Leptactinia leopoldii II Büttner & Nnph. & spont. cult. & Africa & $\mathrm{Cg}$ & med. & $\mathrm{fe}$ \\
\hline \multirow[t]{2}{*}{259} & Sarcocephalus latifolius (Sm.) E. A. Bruce & Mcph. & cult. & Africa & Aftr & med. & Éc.t.r. \\
\hline & Rutaceae (6) & & & & & & \\
\hline
\end{tabular}




\section{Continued}

\begin{tabular}{|c|c|c|c|c|c|c|c|}
\hline 260 & Citrus limon (L.) Burm. f. & Mcph. & cult. & Asia & Pant. & alim. & $\mathrm{fr}$ \\
\hline 261 & Citrus medica L. & Mcph. & cult. & Asia & Pant. & alim. & fr \\
\hline 262 & Citrus $x$ paradisii Macfad. & Mcph. & cult. & Asia & Pant. & alim. & $\mathrm{fr}$ \\
\hline 263 & Citrus reticulata Blanco & Mcph. & cult. & Asia & Pant. & alim. & fr \\
\hline 264 & Citrus sinensis $(\mathrm{L}$.$) Osbeck$ & Mcph. & cult. & Asia & Pant. & alim. med. & Fr. fe \\
\hline \multirow[t]{2}{*}{265} & Ruta chalpensis $\mathrm{L}$. & Nnph. & cult. & Mediterrane & Pant & orn & - \\
\hline & Salicaceae (2) & & & & & & \\
\hline 266 & Flacourtia jangomas (Lour.) Raensch & Mcph. & cult. & Asia & Pant & alim. & Fr. \\
\hline \multirow[t]{2}{*}{267} & Oncoba spinosa Forsk. & Mcph. & cult. & Africa & GC & med. & fe \\
\hline & Scrophulariaceae (2) & & & & & & \\
\hline 268 & Scoparia dulcis L. & Thd. & spont.cult. & Tropical ? & GC & orn & - \\
\hline \multirow[t]{2}{*}{269} & Russelia equisetiformis Schlecht. \& Cham. & Nnph. & cult. & America & Pant & orn & - \\
\hline & Solanaceae (7) & & & & & & \\
\hline 270 & Capsicum frutescens $\mathrm{L}$. & Nnph. & spont. cult. & America & pant & alim. & fr \\
\hline 271 & Datura suaveolens Humb. \& Bonpl. ex Willd. & Nnph. & cult. & America & Pant & orn & - \\
\hline 272 & Nicotiana tabacum L. & Nnph. & cult. & America & & med. & fe \\
\hline 273 & Solanum lycopersicum L. & Thd & cult. & America & Pant. & alim. med. & Fr. fe \\
\hline 274 & Solanum macrocarpon $\mathrm{L}$. & Nnph. & cult. & Africa & Pant & alim. & Fr. \\
\hline 275 & Solanum melongena $\mathrm{L}$. & Chd. & cult. & Asia & Pant & alim. & fr \\
\hline \multirow[t]{2}{*}{276} & Solanum nigrum $\mathrm{L}$. & Chd. & cult. & Eurasia & Pant. & alim. & $\mathrm{fe}$ \\
\hline & Urticaceae (2) & & & & & & \\
\hline 277 & Myrianthus arboreus $\mathrm{P}$. Beauv. & Mcph. & spont. cult. & Africa & CG & alim. med. & Fr. Éc.t \\
\hline \multirow[t]{2}{*}{278} & Pilea microphylla (L.) Liebm. & Thd. & spont.cult. & America & SZ & orn & - \\
\hline & Verbenaceae (2) & & & & & & \\
\hline 279 & Lantana camara L. & Nnph. & spont. cult. & America & Pant. & alim. med. & Fr. fe \\
\hline \multirow[t]{2}{*}{280} & Stachytarpheta jamaicensis (L.) Vahl & Thd. & cult. & America & Pant & orn & - \\
\hline & Vitaceae (1) & & & & & & \\
\hline 281 & Cissus sp. & Gtgr. & spont.cult. & - & Aftr. & alim. & fe \\
\hline
\end{tabular}

Legend: biological types (TB), hemicrytophyte (Hc), mesophanerophyte (Msph), microphanerophyte (Mcph), nanophanerophyte (Nnph), climbing phanerophyte (Phgr), prostrate phanerophyte (Phr), rhizomatous geophyte ( $\mathrm{Gr}$ ), climbing tuberous geophyte (Gtgr), bulbous geophyte (Gb), tuberous geophyte (Gt), erect therophyte (Thd), creeping therophyte (Thr), climbing therophyte (Thgr), erect chamephyte (Chd), creeping chamephyte (Chgr); Status: Spontaneous (spont), cultivated (Cult); Phytogeographic type (TP), Palaeotropical (Paleo), Pantropical (Pant), Cosmopolitan (cosm), Afrotropical (Aftr), AfroMalagasy (Afma), African-American (AfAm), Lower-Guinean (CG), Guineo-Congolese (GC), Sudano-Zambezian (SZ); Usage: Ornamental (orn), medicinal (Med), food (alim); Part use: leaf (fe), rhizome (Rh), meristem (ti), tuber (tub), fruit (fr), stem and/or root bark (Éc. t/r), root (ra), bulb (bulb), stigma (stig), seed (Gr).

4.98\%. It should be noted that $32.47 \%$ of the families have a diversity rate of $0.36 \%$, either one species.

Three biodiversity conservation statuses have been registered in Brazzaville, notably in the Moukonzi-Ngouaka district. Of the 281 taxa: $67.27 \%$ are cultivated and therefore under the close control of the actors; the portion of plants 


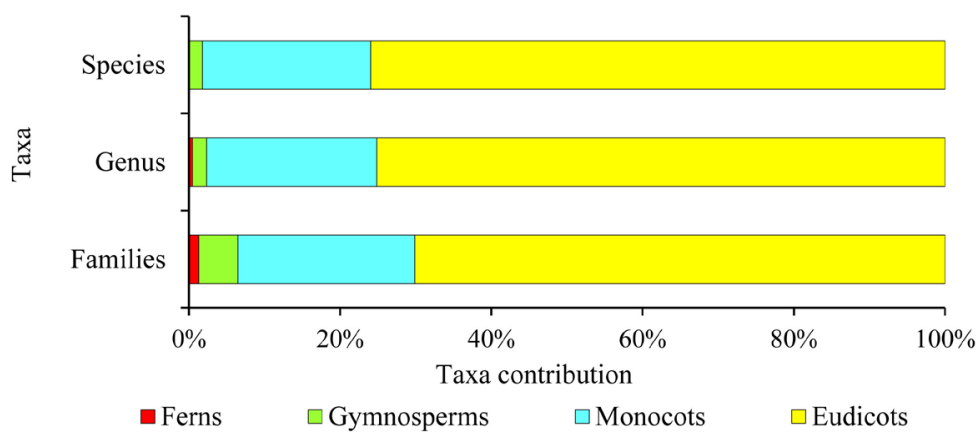

Figure 4. Summary of the taxonomic data of the inventory.

whose naturalization is almost complete but which are still being cultivated is $20.73 \%$; and finally, $12 \%$ of the said taxa are spontaneous, the majority of which are ruderals and nitrophytes. These proportions include introductions from local and foreign migrations.

\subsection{Phytogeographic Distribution}

Phytogeographic data show a clear preponderance of pantropical taxa, which cover more than $2 / 3$ of the inventory (Figure 5). The endemism of the Guinean-Congolese regional centre, constituting the local base element, is poorly represented with less than $1 / 3$ of the taxa inventoried. All other types of phytogeographic distribution, taken as a whole, represent $13.59 \%$. Data on the geographical origin of the taxa show that they originate from three major foci: the Neotropic, notably South and Central America with 33.59\%; the eastern part of the Paleotropic, especially the Asian and Malagasy regions with 21.64\%; and finally, the African portion with $30.60 \%$.

\subsection{Floristic Composition and Natural Habitat}

Of the 281 species recorded, $60.30 \%$ are exotic, many of which are naturalized. The spontaneous cohort of $39.27 \%$ is composed of $3.27 \%$ of the species present in the flora of the Brazzaville Patte d'Oie forest, $18.54 \%$ of ruderal taxa and nitrophytes, and finally the 2 main Congolese ecosystems. The savannas, in addition to those of the Léfini phytogeographic district, contribute $3.27 \%$. The dense humid forest underpins this flora at $14.18 \%$.

This census highlights four taxa, including three non-natives (Mangifera indica, Persea americana, Carica papaya) and one native (Dacryodes edulis) widely cultivated. The parcel preponderance of each taxon varies on average from $1 / 3$ to $1 / 2$. Ornamentals, without any other use, are less prized and their presence seems to be a fad or just a snobbery of the populations.

\subsection{Personalized Interview Data}

The interviews focused specifically on the reasons for introducing and/or cultivating these plants in their parcel. Ninety-one percent of the survey revealed that this practice is underpinned by the satisfaction of 1) socio-cultural needs 


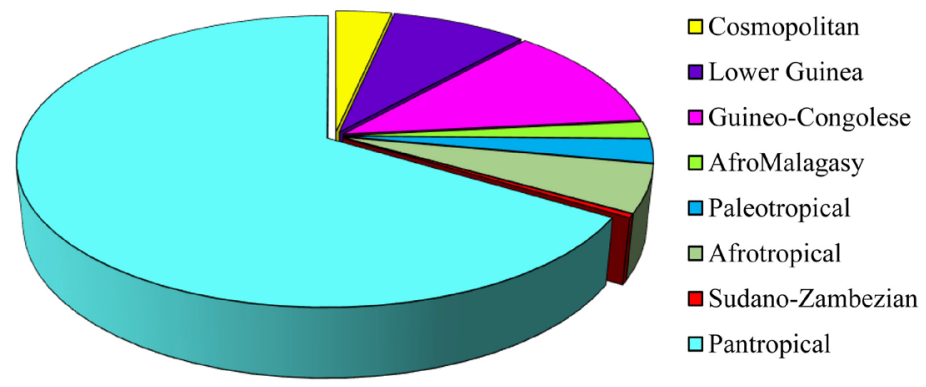

Figure 5. Synoptic of the phytogeographic distribution of taxa.

(traditional medicine, cultuals, etc.) and 2) food needs. Taking into account the totality of the data, $79.56 \%$ of the informants favored the food aspect, $89.92 \%$ traditional medicine and cultuals, and $22.34 \%$ the aesthetic aspect.

The socio-cultural benefits, without being exhaustive, are provided by savannah taxa such as Bridelia ferruginea, Sarcocephalus latifolius, Millettia versicolor... and forest taxa such as Trilepisium madagascariense, Dioscorea spp., Acanthus montanus, Lannea welwitschii, Canarium schweinfurthii, Buchholzia macrophylla... As for food requirements, we can mention Elaeis guineensis, Trilepisium madagascariense, Canarium schweinfurthii, Glyphaea brevis, Cola spp...

Finally, in spite of the local non-native species of the flora of Brazzaville, for the satisfaction of these needs, the populations use naturalized taxa such as Mangifera indica, Persea americana, Sambucus mexicana, Spondias spp., Annona spp., polyscias spp., Carica papaya, Eugenia uniflora...

\subsection{Ecosystem Service Data}

The benefits resulting from the use of this flora are capitalized in three well-hierarchical categories. Traditional phytotherapy is favored by $89.83 \%$ of the respondents, while nutrition follows with $79.56 \%$ and, finally, aesthetics with $22.33 \%$. Informants did not express an opinion on all other services, which received $0 \%$.

Data on the use of taxa divided them into two groups: 1) those specific to one of the 3 categories, and 2) those with multiple uses. Multiple-use taxa occur concomitantly in either category. For example, ornamental plants, which cover $48.36 \%$ of the taxa, are simultaneously involved in the medicinal aspect to the extent of $4 \%$. This is also true for food species whose contribution is $28.36 \%$, with a traditional medicine intervention rate of $8 \%$. Finally, the medicinal cohort, with $35.27 \%$ of taxa, is involved in feeding and ornamentation (Figure 6). It should be noted that multiple-use species are the most sought-after and maintained by the population.

These floristic data on uses highlight two categories of ecosystem services which are (1) harvesting services with food and traditional medicine, (2) and socio-cultural with the aesthetic value of ornamental plants. In addition to these palpable aspects, populations indirectly exploit other ecosystem services. The 


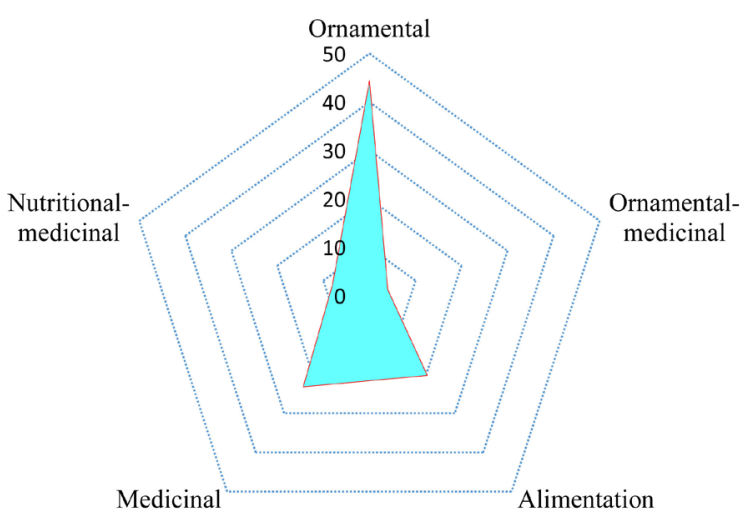

Figure 6. Expressed ethnobotanical value of the inventoried flora (\%).

fact that their benefits are seasonal and/or occasional, or even intangible, means that their influence has less impact on people. The benefit-based floristic analysis shows that multiple-use species are well represented in numbers, but not in terms of individuals per taxon. These taxa cover all categories and several ecosystem services such as food, traditional therapy, handicrafts, shade, shelterbelts, income generation, fuelwood, spiritual well-being and aesthetics. The species used are Dacryodes edulis, Mangifera indica, Persea americana, Elaeis guineensis, Buchholzia macrophylla, Gardenia jovis-tonantis, Polyscia spp., Euphorbia spp., canarium schweinfurthii...

\subsubsection{Supplying Services}

Food anthropology and traditional medicine are the most cited use values. These two aspects, which mobilize $64 \%$ of the inventory, constitute the fundamental source of the introduction of plants to the Moukonzi-Ngouaka district in Brazzaville.

The use of organs is associated with traditional phytotherapy and food anthropology (Figure 7). Vegetative organs are the most popular with $52.31 \%$, of which leaves provide $33.45 \%$. As for the generative organs, the overall contribution amounts to $19.57 \%$ and fruits cover the bulk of the contribution at $18.50 \%$. The dominance of leaves and fruits is explained by the fact that these organs are strongly involved in traditional food and medicine. While the Zea mays stigmas, roots and bark of various species are specific to traditional herbal medicine, all other organs satisfy both ecosystem services equally. These two fundamental aspects of the relationship between people and biodiversity overshadow income generation, fuelwood, shelterbelt and climate regulation, which would be classified as secondary functions.

\section{1) Food anthropology}

Food plants, which make up $28.36 \%$ of the flora, are mostly naturalized taxa. The spontaneous flora introduced in Brazzaville contributes 11.74\%. According to morphological types, this fraction is as follows: trees (27.16\%), shrubs $(19.75 \%)$, grasses $(41.98 \%)$, and lianas (11.11\%). The most prized organs are fruits and leaves (Figure 8). 


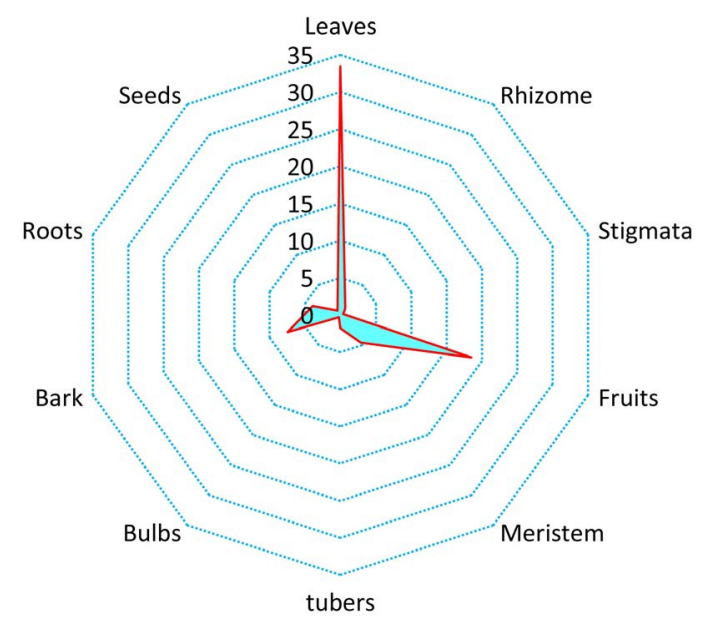

Figure 7. Synoptic overview of organs used by the population (\%).

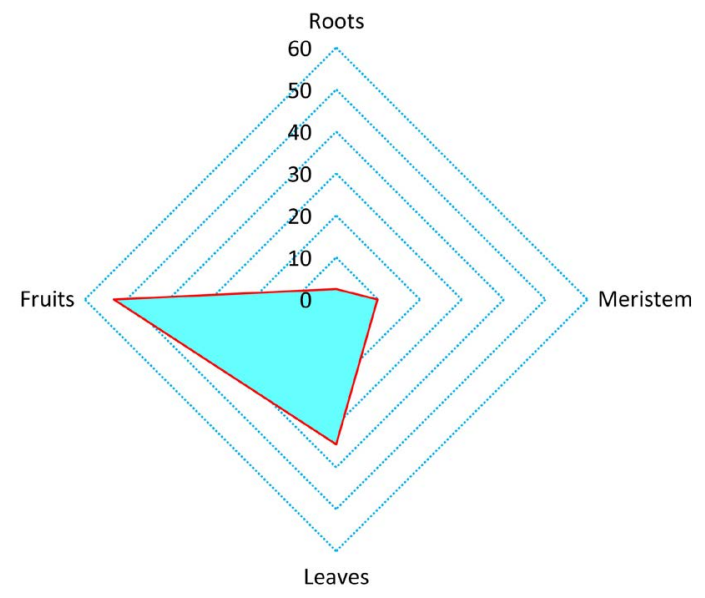

Figure 8. Contribution of food species through useful organs (\%).

\section{2) Traditional phytotherapy}

The medicinal flora represents $35.27 \%$ of the inventory and covers all morphological types. The contribution of spontaneous flora amounts to $22.06 \%$, the majority of which comes from outside the phytogeographical district of Léfini. Arborescent species are clearly predominant (64.44\%) compared to herbaceous (31.11\%) and lianas (4.44\%). Leaves (57.94\%) and stems (19.62\%) are the most sought-after organs (Figure 9).

\subsubsection{Socio-Cultural Services}

Ornamental flora accounts for $48.36 \%$ of the taxa recorded (Figure 10). Tree species (60.48\%) dominate herbaceous (37.10\%) and lianas (2.42\%). These plants are found as components of quickset hedges (Clerodendrum inerme, Pedilanthus tithymaloides, Newbouldia laevis, Dracaena spp, Gymnanthemum amygdalinum, Quisqualis indica, Antigonum leptopus, Thunbergia erecta, Aristolochia sp....); delimiting the concessions, in the form of a small flowered garden (Portulaca oleracea, Ixora coccinea, Clerodendrum splendens, Alpinia spp., Zingiber officinale, Russelia equisetiformis, Mirabilis jalapa, Acalypha spp...); 


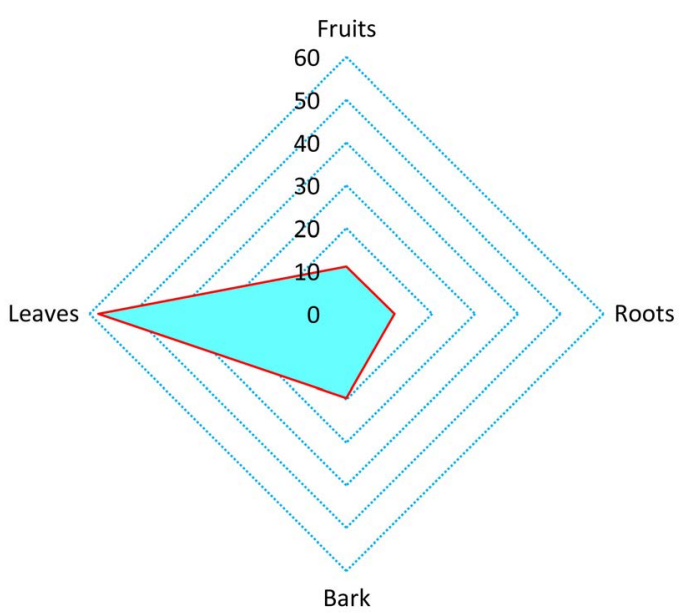

Figure 9. Contribution of therapeutic species through useful organs (\%).

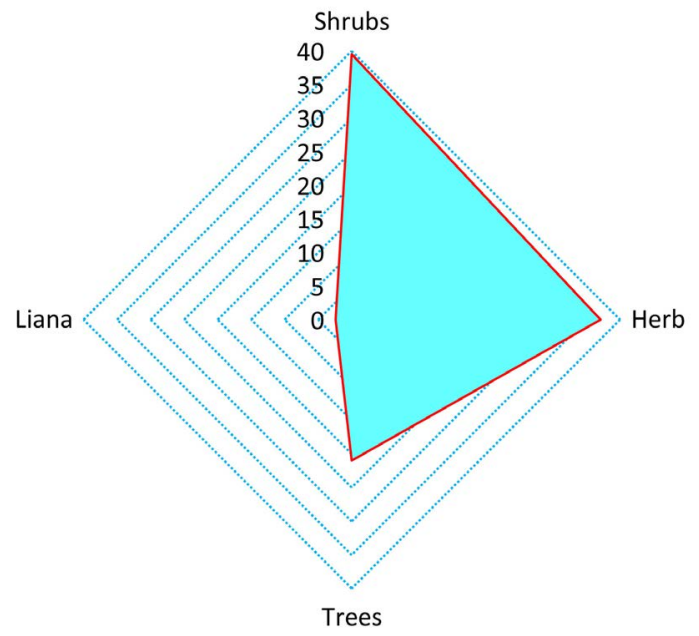

Figure 10. Distribution of ornamental plants by morphological type (\%).

isolated individual and if necessary just shade (Lannea welwitschii, Cananga odorata, Spondias dulcis, Mangifera indica, Persea americana, Canarium schweinfurthii, Cerus peruvianus, Buchholzia macrophylla, Erythrophleum suaveolens, Peltophorum pterocarpum, Pentaclethra eetveldeana, Artocarpus altilis, Trilepisium madagascariense, Myrianthus arboreus...).

\section{Discussion}

\subsection{Plants and Human Transhumance}

The history of humankind is made up of encounters and exchanges of all kinds, including plants useful in more than one way [1] [9] [10] [11] [12] [13] [56]. As a result of continental drift, the new centres of differentiated floristic speciation in the new territories have experienced a floristic explosion whose taxa are spread throughout the rest of the world [6] [7] [8]. Initially confined to new territories, these taxa have overcome adaptation and naturalization mechanisms to the point of competing with native flora [14] [62] [63] [64]. The history of plant 
migration throughout the world is deeply coupled with that of man and these taxa provide evidence of the economic and cultural factors that govern their selection [6]. The colonial period was marked by a process of massive introduction of species into the "new territories", based on an aesthetic affectation, the creation of botanical gardens, supply points for slave ships, and the establishment of colonial plantations, especially cash crops [5] [6] [7]. Exchanges between phytogeographical regions of the world, at any scale, generate floristic changes [5] [8]. Notwithstanding the exchanges at the level of empires and regions, those that take place within the territories of a region are not negligible. These very small-scale exchanges are generally based on rural exodus, the corollary of which is impoverishment. The difficulty of satisfying primary needs on a daily basis is such that populations resort to the values of their socio-cultural base. This phenomenon explains the introduction of several taxa from one phytogeographical district to another. In the present case, the taxa Bridelia ferruginea, Millettia versicolor, Gardenia jovis-tonantis, Hymenocardia acida, Trilepisium madagascariense, Myrianthus arboreus, Mondia whitei, Cola acuminata... are a tangible example of very low-level exchange and perpetuation of the values of the socio-cultural base.

\subsection{Humans and Activities}

Like the majority of sub-Saharan cities, especially working-class neighborhoods, Moukonzi-Ngouaka is home to an exhdered population, most of whom live below the poverty line of less than US\$1 a day [65]. Indeed, the majority of the population works in the informal sector, for financial satisfaction. The disorganization of this sector is such that it is equivalent to daily subsistence. The corollaries are the impoverishment of society, especially of the most vulnerable social stratum, which cannot meet basic food and health needs due to prohibitive costs [15] [18] [66] [67]. Faced with this reality, the population most often resorts to traditional knowledge to meet basic needs, as several studies have noted [18] [19] [20] [66]. As the use of traditional knowledge is linked to useful plants (food and phytotherapeutic), the massive presence of non-native species with proven medicinal and dietary properties in Moukonzi-Ngouaka is nothing other than a result of internal migration or even the rural exodus of populations [67] [68]. This duality authenticates the existence of savannah taxa (Bridelia ferruginea, Sarcocephalus latifolius, Millettia versicolor, Hymenocardia acida, Gardenia jovis-tonantis, Elaeis guineensis...), forest taxa (Mondia whitei, Myrianthus arboreus, Trilepisium madagascariense, Canarium schweinfurthii, Terminalia superba, Cola acuminata, Buchholzia macrophylla, Markhamia tomentosa, Caloncoba welwitschii, Dioscorea spp...) and wetlands, both savannah and forest ( $C_{y}$ perus articulatus, Costus afer, Brillantaisia patula, Acanthus montanus...).

An analysis of data from the traditional African and particularly Congolese pharmacopoeia points to the use of several of these plants for the same purposes in their geographical area [2] [62] [69] [70] [71]. This universal value associated 
with plants is maintained by the displacement of populations, which is the origin of exchanges and the mixing of knowledge. This phenomenon is said to be the main source of use of non-native species outside their natural range [2] [4] [14]. Without being exhaustive, the taxa referenced in this study are Peltophorum pterocarpum, Senna siamea, Annona muricata, Eucalyptus spp., Eugenia uniflora, Psidium guayava, Hura crepitans, Jatropha curcas...

\subsection{Arboriculture and Ecosystem Services}

Ecosystems of all types and compositions provide humans and humanity with a variety of benefits, expressed in terms of ecosystem goods and services [27] [28]. This value is expressed in tangible, and therefore palpable, or intangible ways and is a foundation for the sustainability of ecosystems and the social and economic well-being of human populations [72] [73] [74]. In addition to the above-mentioned aspects, the integration of exotic taxa into the local flora has beneficial effects, equivalent to added value [75] [76] [77]. Plants provide direct benefits to the population (food security, artisanal material, fuelwood, source of income and phytopharmacognosia) and indirect benefits (climate regulation, carbon sequestration, pollution detection, dust sequestration, etc.) to the community [78] [79] [80].

The analysis of use data coupled with the ecosystem categories and services shows harvesting with food and phytotherapy as ecosystem services, and socio-cultural with aesthetic value. No other services, direct or indirect, particularly regulation services, are included in the benefits derived from arboriculture, the basis of urban forestry [14] [81] [82] [83]. The population's vision on the introduction of plants into their environment is mainly influenced by practices rooted in traditional knowledge. Daily observation shows that urban arboriculture represents a non-negligible source of fuelwood and income for the most vulnerable populations. In spite of this recognized value, the fact that it is seasonal and occasional would overshadow its importance.

\subsection{Direct Values and Benefits of Biodiversity}

Urban populations living far from forests would be expected to be less impacted by ecosystem services [84] [85]. As the city of Brazzaville has developed on a formerly marshy terrain dominated by a savannah plateau and mesophilic forests, landscape modelling has accompanied its development [15]. An analysis of the current flora shows that the evolution of plant cover has been to the detriment of spontaneous biodiversity. As the introduction of these plants is intimately linked to human history, the inventory reveals a disparity in the floristic composition from one parcel to another. Except for the taxa involved in the delimitation of properties as living hedges, which are more or less constant, all the others reflect the socio-cultural base of the inhabitants and, better still, the endogenous knowledge that lies dormant in each of them [85] [86]. This environment thus constituted and resulting from the exploitation of the value of biodiversity, associated with the socio-cultural bases, would correspond to the limit of 
a socio-ecosystem [87] [88] [89] [90]. The anthropogenic floristic cohort is involved in two categories of ecosystem services, either harvesting or socio-cultural. In harvesting, the focus is on food aspects and traditional phytotherapy, whereas aesthetics is the only ecosystem service recognised in the socio-cultural category. The benefits generated by these ecosystem services are such that they obscure the income from the sale of the products of these plants, especially the fruits. This consideration would arise from the fact that the seasonality of these revenues, although additional and subsequent, does not override the daily phytotherapeutic and food values. Finally, since the values expressed by populations on ecosystem services are based on Non-Timber Forest Products (NTFPs), urban forestry, notwithstanding its ecological functions, can be a lever for poverty alleviation [91] [92] [93] [94]. From a harvesting and socio-cultural point of view, let us recognize the place of wood energy and the source of income, two elements fundamental to the survival provided by urban arboriculture as a unit of socio-ecological systems [92] [95] [96].

\subsection{Indirect Values and Benefits of Biodiversity}

Urban forestry, of which arboriculture is the major pillar, provides functions that are essential to the proper functioning of the city and even of humanity, all things considered [97]. As urban cities are essential sources of heat island emissions, plants and especially woody plants sequester carbon and release oxygen through physiological metabolic processes [64] [80] [98]. Through these mechanisms, plants effectively combat urban heat islands while maintaining human well-being, air quality and of course zonal or even regional climate regulation [79] [98] [99] [100].

Urban arboriculture could also have positive effects on health, in a city where the population has less than $0.5 \mathrm{~m}^{2}$ of green space per person [98] [101]. Notwithstanding the success of unspoken intangible phenomena, people derive benefits from plants, described as secondary, such as shade, windbreak role and spiritual well-being. In addition to these, several others, more subtle and fundamental, organize their daily existence [74] [100] [102].

\subsection{Methodological Achievements}

In research, all methods are valid under two conditions: 1) clearly identify the objective; 2) marginalize subjective factors. The different stages of the study have led to distinguishing 1) native taxa from exotics by analysing speciation centers; 2 ) the knowledge acquired from the mixing of knowledge for local populations; 3) to understand the benefits derived from this double introduction of plants and the reasons for which they are introduced; 4) the perpetuation of the socio-cultural base is the primary unit that feeds it via internal migration; 5) finally, floristic variability is a function of individual or even clan knowledge. In addition to this highlighted material aspect, the immaterial aspects of urban arboriculture are not lagging behind. This part of the research highlights the gap between the actors on the benefits generated by urban arboriculture and its exploitation. 


\section{Conclusion}

The floristic enrichment that has profoundly modified the urban ecosystem of Brazzaville, although continuing very actively, has gone through two phases and two groups of actors. The colonial period marked by a massive intrusion of exotic species was relayed by internal migrations, responsible for contributions that enhanced the socio-cultural foundation. The exotic flora being integrated as a local component by the populations obscured the share that is not negligible of the contributions induced by internal migration. From the mixing of customs and exchanges, the populations have increased the socio-cultural background and efficiently exploit more floristic units, for the daily satisfaction of basic needs (material and immaterial). Arboriculture, a fundamental facies of urban forestry, provides multiple functions that guarantee a pleasant life in the city. Generally maintained with the aim of having at hand the source of materials indispensable for socio-cultural uses, urban forestry is a sure value for the conservation of biodiversity. In this particular ecosystem, several ecosystem services interact intelligently through multiple-use plants for the well-being of populations. Finally, the value of ecosystem services is not a panacea exclusive to large forest massifs and their impact is limited to riparian populations, but rather associated with all plant formations, regardless of their immensity and floristic composition.

\section{Conflicts of Interest}

The authors declare no conflicts of interest regarding the publication of this paper.

\section{References}

[1] Chevalier, A. (1931) Le rôle de l'Homme dans la dispersion des plantes tropicales. Échanges d'espèces entre l'Afrique Tropicale et l'Amérique du Sud. Revue de botanique appliquée et d' agriculture coloniale, 120, 633-650. https://www.persee.fr/doc/jatba 0370-3681_1931_num $111120 \quad 5016$ https://doi.org/10.3406/jatba.1931.5016

[2] Raponda-Walker, A. and Sillans, R. (1961) Les plantes utiles du Gabon. Sépia, France. https://doi.org/10.3109/13880206109066644

[3] Haeringer, P. (1980) L'arbre dans la ville: lecture sociale en quatre tableaux du couvert végétal dans la ville africaine. Cahier ORSTOM, Sér. Sciences Humaine, 17, 289-308.

[4] Villapoll, N. (1984) Les habitudes alimentaires africaines en Amérique latine, In: L’Afrique en Amérique latine (Le monde en Amérique latine). Unesco, Paris.

[5] Devineau, J.-L. and Guillaumet, J.-L. (1992) Origine, nature et conservation des milieux naturels africains: Le point de vue des botanistes. Afrique contemporaine, 161, 79-90.

[6] Fleury, M. (1994) Impact de la traite des esclaves sur la phytogéographie. Exemple chez les Aluku (Boni) de Guyane française. Journal d Agriculture Traditionnelle et de Botanique Appliquée, 36, 113-134. https://doi.org/10.3406/jatba.1994.3537

[7] Katz, E. (1994) Du mûrier au caféier: Histoire des plantes introduites en pays 
mixtèque (xvi- $\mathrm{xx}^{\mathrm{e}}$ siècle). Journal d Agriculture Traditionnelle et de Botanique Appliquée, 36, 209-244. https://doi.org/10.3406/jatba.1994.3541

[8] Puig, H. (1994) Relations floristiques amphi-néotropicales: les cas du Mexique et des Guyanes. Mémoire de la Société de Biogéographie, 4, 21-36.

[9] Collin Delavaud, C. (2000) Plantes européennes introduites au Pérou. In: Les plantes de l'Ancien monde à la conquête de l'Amérique latine. Cahiers d'outre-mer, 209-210, 111-128. https://doi.org/10.3406/caoum.2000.3760 https://www.persee.fr/doc/caoum 0373-5834 2000 num $53 \quad 209 \quad 3760$

[10] Huetz de Lemps, A. (2000) Introduction: La conquête de l'Amérique latine par les plantes de l'Ancien Monde. In: Les plantes de l'Ancien monde à la conquête de l'Amérique latine. Cahiers d Outre-mer, 209-210, 2-6.

https://www.persee.fr/doc/caoum 0373-5834 2000 num 532093755

[11] Huetz de Lemps, A. (2000) Principales plantes cultivées introduites en Amérique latine depuis 1492. In: Les plantes de l'Ancien monde à la conquête de l'Amérique latine. Cahiers d Outre-mer, 209-210, 129-186.

https://www.persee.fr/doc/caoum 0373-5834 2000 num $53 \quad 2093761$

https://doi.org/10.3406/caoum.2000.3761

[12] Maillard, J.-C. (2000) Une greffe réussie en terre américaine, ou comment le bananier a conquis le Nouveau Monde. In: Les plantes de l'Ancien monde à la conquête de l'Amérique latine. Cahiers d' Outre-mer, 209-210, 7-30.

https://www.persee.fr/doc/caoum 0373-5834 2000 num $53 \quad 209 \quad 3756$ https://doi.org/10.3406/caoum.2000.3756

[13] Péhaut, Y. (2000) Le cocotier et le palmier à huile en Amérique. In: Les plantes de l'Ancien monde à la conquête de l'Amérique latine. Cahiers d'Outre-mer, 209-210, 55-94. https://www.persee.fr/doc/caoum 0373-5834 2000 num $53 \quad 2093758$ https://doi.org/10.3406/caoum.2000.3756

[14] Kimpouni, V., Mbouba, S.D. and Motom, M. (2017) Étude de la flore allochtone arborescente et foresterie urbaine à Brazzaville (Congo). Journal de Botanique Société Botanique de France, 79, 73-92.

[15] Kimpouni, V., Mamboueni, J.C., Lenga-Sacadura, M.-Y. and Nsika Mikoko, E. (2017) Recipes and Treatments in Traditional Herbal Medicine to the Kaamba Community of Madingou, Congo. European Journal of Medicinal Plants, 20, 1-13. https://doi.org/10.9734/EJMP/2017/35096

[16] Lambinon, J. (2000) Les introductions de plantes non indigènes dans l'environnement naturel. Edition Conseil de l'Europe, Bruxelles.

[17] Vanderhoeven, S., Branquart, E., Grégoire, J.-C. and Mahy, G. (2007) Les espèces exotiques envahissantes. Dossier scientifique réalisé dans le cadre de l'élaboration du Rapport analytique 2006-2007 sur l'état de l'environnement wallon. http://environnement.wallonie.be/eew

[18] Kimpouni, V., Lenga-Sacadura, M.-Y., Mamboueni, J.C. and Nsika Mikoko, E. (2018) Phytodiversité et pharmacopée traditionnelle de la communauté Kaamba de Madingou (Bouenza-Congo). European Scientific Journal, 14, 191-220.

https://doi.org/10.19044/esj.2018.v14n3p191

[19] Kimpouni, V., Mamboueni, J.C., Mboussy Tsoungould, F.G. and Nsika Mikoko, E. (2019) Ethnobotanical and Phytotherapeutic Study from Kouni Community of the Sub-Prefecture of Kayes (Bouenza-Congo). Heliyon, 5, 1-11.

https://doi.org/10.1016/j.heliyon.2019.e02007

[20] Kimpouni, V., Mamboueni, J.C., Mboussy Tsoungould, F.G. and Nsika Mikoko, E. (2019) Environment and Livelihood of the Kouni Community of the Kayes 
Sub-Prefecture (Bouenza, Congo). Ethnobotany Research and Applications, 18, 1-15. https://doi.org/10.32859/era.18.44.1-15

[21] Makany, L. (1976) Végétation des plateaux Téké (Congo). Collection Travaux Université de Brazzaville. Université de Brazzaville, Brazzaville.

[22] Kimpouni, V., Mbou, P., Apani, E. and Motom, M. (2013) Floristic Diversity and Structural Parameters of the Brazzaville Patte d'Oie Forest, Congo. Open Journal of Ecology, 3, 518-531. https://doi.org/10.4236/oje.2013.38061

[23] Kimpouni, V., Mbou, P., Gakosso, G. and Motom, M. (2013) Biodiversité floristique du sous-bois et régénération naturelle de la forêt de la Patte d'Oie de Brazzaville, Congo. International Journal of Biological and Chemical Sciences, 7, 1255-1270. https://doi.org/10.4314/ijbcs.v7i3.31

[24] Kimpouni, V., Apani, E., Mbou, P. and Motom, M. (2014) Étude floristique des îlots forestiers naturels de la Patte d'Oie de Brazzaville, Congo. Acta Botanica Gallica, 161, 63-79. https://doi.org/10.1080/12538078.2013.870048

[25] Kuchelmeister, G. (2000) Des arbres pour le millénaire urbain: Le point sur la foresterie urbaine. Unasylva, 200, 49-55.

[26] Nzala, D. and Miankodila, P. (2002) Arbres et espaces verts à Brazzaville (Congo). Bois et forêts des tropiques, 272, 88-92.

[27] Diatta, A.A., Ndour, N., Manga, A., Sambou, B., Faye, C.S., Diatta, L., Goudiaby, A., Mbow, C. and Dieng, S.D. (2016) Services écosystémiques du parc agroforestier à Cordyla pinnata (Lepr. ex A. Rich.) Milne-Redh. Dans le Sud du Bassin Arachidier (Sénégal). International Journal of Biological and Chemical Sciences, 10, 2511-2525. https://doi.org/10.4314/ijbcs.v10i6.9

[28] Dieng, S.D., Diop, M., Goudiaby, A., Niang-Diop, F., Faye, L.C., Guiro, I., Sambou, S., Lykke, A.M. and Sambou, B. (2016) Caractérisation des services écosystémiques fournis par Cordyla pinnata dans la périphérie de la Forêt classée de Patako au Sénégal. Vertig-la revue électronique en sciences de Penvironnement [En ligne], 16, 41-49. http://journals.openedition.org/vertigo/17634 https://doi.org/10.4000/vertigo.17634

[29] Koussibila-Dibansa, G.M. and Makaya, D. (1997) Contribution à l'étude de la flore des jardins de cases de Brazzaville: Cas de Moukoundzi-Ngouaka. Mém. CAPES Sc. Nat., Université Marien Ngouabi, Brazzaville.

[30] Samba-Kimbata, M.-J. (1978) Le climat Bas congolais. Thèse de doctorat, Université de Dijon, Dijon.

[31] Vennetier, P. (1977) Atlas de la République Populaire du Congo. Édition Jeune Afrique, Paris.

[32] Köppen, W. (1900) Versuch einer Klassifikation der Klimate, vorzugsweise nach ihren Beziehungen zur Pflanzenwelt. Geographische Zeitschrift, 6, 593-679.

[33] Köppen, W. (1936) Das geographische System der Klimate. Gebrüder Borntraeger, Berlin.

[34] Kottek, M., Grieser, J., Beck, C., Rudolf, B. and Rubel, F. (2006) World Map of the Köppen-Geiger Climate Classification Updated. Meteorologische Zeitschrift, 15, 259-263. https://doi.org/10.1127/0941-2948/2006/0130

[35] Peel, M.C., Finlayson, B.L. and McMahon, T.A. (2007) Updated World Map of the Köppen-Geiger Climate Classification. Hydrology and Earth System Sciences, 11, 1633-1644. http://www.hydrol-earth-syst-sci.net/11/1633/2007/ https://doi.org/10.5194/hess-11-1633-2007

[36] Belda, M., Holtanová, E., Halenka, T. and Kalvová, J. (2014) Climate Classification 
Revisited: From Köppen to Trewartha. Climate Research, 59, 1-13. https://doi.org/10.3354/cr01204

[37] Beck, H.E., Zimmermann, N.E., McVicar, T.R., Vergopolan, N., Berg, A. and Wood, E.F. (2018) Data Descriptor: Present and Future Köppen-Geiger Climate Classification Maps at 1-km Resolution. Scientific Data, 5, Article ID: 180214. https://doi.org/10.1038/sdata.2018.214

[38] Samba, G. and Nganga, D. (2011) Rainfall Variability in Congo-Brazzaville: 1932-2007. International Journal of Climatology, 32, 854-873. https://doi.org/10.1002/joc.2311

[39] Samba, G., Nganga, D. and Mpounza, M. (2008) Rainfall and Temperature Variations over Congo-Brazzaville, between 1950 and 1998. Theoretical and Applied Climatology, 91, 85-97. https://doi.org/10.1007/s00704-007-0298-0

[40] Olson, D.M., Dinerstein, E., Wikramanayake, E.D., Burgess, N.D., Powell, G.V.N., Underwood, E.C., D’amico, J.A., Itoua, I., Strand, H.E., Morrison, J.C., Loucks, C.J., Allnutt, T.F., Ricketts, T.H., Kura, Y., Lamoreux, J.F., Wettengel, W.W., Hedao, P. and Kassem, K.R. (2001) Terrestrial Ecoregions of the World: A New Map of Life on earth. BioScience, 5, 933-938. https://doi.org/10.1641/0006-3568(2001)051[0933:TEOTWA]2.0.CO;2

[41] White, F. (1983) The Vegetation of Africa: A Descriptive Memoir to Accompany the Unesco/AETFAT/UNSO Vegetation Map of Africa. Unesco, Paris.

[42] Kimpouni, V., Lejoly, J. and Lisowski, S. (1992) Les Eriocaulaceae du Congo. Fragmenta Floristica Geobotanica, 37, 127-145.

[43] Denis, B. (1970) Les sols de la région de Brazzaville. Orstom, Brazzaville.

[44] Pauwels, L. (1993) Nzayilu N’ti: Guide des arbres et arbustes de la région de Kinshasa-Brazzaville. Jardin Botanique National de Belgique, Meise.

[45] Sita, P. and Moutsambote, J.-M. (1988) Catalogue des plantes vasculaires du Congo. CERVE/Orstom, Brazzaville.

[46] Graf, A.B. (1992) Hortica: Color Cyclopedia of Garden Flora and Indoor Plants. Roehrs Co., UK.

[47] Graf, A.B. (2003) Tropica: Color Cyclopedia of Exotic Plants and Trees for Warm-Region Horticulture in Cool Climate the Summer Garden of Sheltered Indoors. 4th Edition, Revised and Enlarged, Roehrs Co, UK.

[48] Chih Min, B., Omar-Hor, K. and Chow Lin, O.-Y. (2006) 1001 Garden Plants in Singapore. 2nd Edition, 5th Print, National Parks' Publication, Singapore.

[49] APG (2016) An Update of the Angiosperm Phylogeny Group Classification for the Orders and Families of Flowering Plants: APG IV. Botanical Journal of the Linnean Society, 181, 1-20. https://doi.org/10.1111/boj.12385

[50] PPG (2016) A Community-Derived Classification for Extant Lycophytes and Ferns: Pteridophyte Phylogeny Group (PPG I). Journal of Sytematics and Evolution, 54, 563-603. https://doi.org/10.1111/jse.12229

[51] Lebrun, J.-P. and Stork, A.L. (1991-2015) Enumeration of Flowering Plants in Tropical Africa and Tropical African Flowering Plants: Ecology and Distribution. 1-10, Published by the Conservatory and Botanical Garden of the City of Geneva, Geneva. http://www.villege.ch/musinfo/bd/cjb/africa/recherche.php?langue=en

[52] Kimpouni, V., Nzila, J.D.D. and Kaya H.F. (2019) Urban Forestry and Ecosystem Services in the City of Dolisie (Congo). American Journal of Agriculture and Forestry, 7, 53-65. https://doi.org/10.11648/j.ajaf.20190702.13

[53] Mbouba, S.D. (2007) Importance et aspects biogéographiques de la flore ligneuse 
allochtone à Brazzaville. Mém. CAPES Sc. Nat., Université Marien Ngouabi, Brazzaville.

[54] White, F. (1979) The Guineo-Congolian Region and Its Relationships to Other Phytochoria. Bulletin du Jardin Botanique National de Belgique, 49, 11-55. https://doi.org/10.2307/3667815

[55] Schnell, R. (1971) Introduction à la phytogéographie des pays tropicaux. 1-Les flores, les structures. Gauthier-Villars, Paris.

[56] Vanden Berghen, C. (1982) Initiation à l'étude de la végétation. $3^{e}$ édition, Jardin Botanique de Belgique, Meise.

[57] Letouzey, R. (1982-1983) Manuel de botanique forestière: Afrique tropicale. CTFT, Nogent-sur-Marne.

[58] Raunkiaer, C. (1934) The Life Forms of Plants and statistical Plant Geography. Clarendon Press Oxford.

[59] Lebrun, J. (1964) À propos des formes biologiques des végétaux en régions tropicales. Bulletin de I Académie Royale do Outre-Mer, 4, 926-937.

[60] Martin, G.J. (1995) Ethnobotany: A Methods Manual. Chapman \& Hall, London.

[61] Grenand, P. and Prévost, M.-F. (1994) Les plantes colorantes utilisées en Guyane française. Journal d'Agriculture Traditionnelle et de Botanique Appliquée (nvlle série), 36, 139-172. https://doi.org/10.3406/jatba.1994.3538

[62] Kimpouni, V. and Motom, M. (2006) Note sur Inga edulis (Leguminosae) au Congo Brazzaville. Systematics and Geography of Plants, 76, 245-250.

[63] Lacoste, A. and Salanon, R. (2006) Éléments de biogéographie et d'écologie: une compréhension de la biosphère par l'analyse des composantes majeures des écosystèmes. $2^{\mathrm{e}}$ Edition, Armand Colin, Paris.

[64] Kimpouni, V., Apani, E., Kouvoukana, V. and Motom M. (2012) Variation de la composition floristique des fruitiers traditionnels après abandon en zone de savane. Acta Botanica Gallica, 159, 53-65. https://doi.org/10.1080/12538078.2012.671643

[65] CNSEE (2010) Recensement Général de la Population et de l'Habitat de 2007; en Quelques Chiffres, MEPATI, UNFPA, MEPATI, Brazzaville.

[66] WHO (2002) Traditional Medicine Strategy 2002-2005. http://www.who.int/medicines/library/trm trat eng.pdf

[67] Badiaga, M. (2011) Etude ethnobotanique, phytochimique et activités biologiques de Nauclea latifolia Smith, une plante médicinale africaine récoltée au Mali. Thèse de doctorat, Université Blaise Pascal, Clermont-Ferrand.

[68] Nsonde Ntandou, G.F., Kimpouni, V., Dianzitoukoulou Matsima, L.D. and Abena, A.A. (2020) Evaluation of Antipyretic and Analgesic Effects of Alchornea cordifolia Schum. \& Thonn. (Euphorbiaceae) and Quassia Africana Baill (Simaroubaceae). International Journal of Sciences, 9, 1-7. https://doi.org/10.18483/ijSci.2255

[69] Bouquet, A. (1969) Féticheurs et médecines traditionnelles du Congo (Brazzaville). Mém. ORSTOM n 36, Orstom, Brazzaville.

[70] Adjanohoun, E.J., Ahyi, A.M.R., Ake Asi, L., Baniakina, J., Chibon, P., Cusset, G., Doulou, V., Enzanza, A., Eyme, J., Goudote, E., Keita, E., Mbemba, C., Mollet, J., Moutsamboté, J.-M., Mpati, J. and Sita P. (1988) Contribution aux études ethnobotaniques et floristiques en République Populaire du Congo: Médecine traditionnelle et pharmacopée. ACCT, Paris.

[71] Sofowara, A. (1996) Plantes Médicinales et Médecine Traditionnelle d’Afrique. Karthala, Paris.

[72] Millenium Ecosystem Assessment (MEA) (2003) Ecosystems and Human Well-Being: 
A Framework for Assessment. Island Press, Washington DC.

[73] Millenium Ecosystem Assessment (MEA) (2005) Ecosystems and Human Well-Being: Synthesis. Island Press, Washington DC.

[74] Roche, P., Geijzendorffer, I., Levrel, H. and Maris, V. (2016) Valeurs de la biodiversité et services écosystémiques: Perspectives interdisciplinaires. Collection Update Sciences \& Technologies. Editions Quæ, Paris. https://doi.org/10.3917/quae.roche.2016.01

[75] Kuchelmeister, G. and Braatz, S. (1993) Nouveau regard sur la foresterie urbaine. Unasylva, 173, 3-12.

[76] Ozenda, P. (1982) Les végétaux dans la biosphère. Doin, Paris.

[77] Convery, F.J. (1990) Intégration d'un processus de plan national d'action pour l'environnement dans le cadre macro-économique. In: Plans nationaux d action pour Penvironnement en Afrique. Compte rendu d'atelier (Dublin, Irlande) du 13-14 décembre 1990), Gouvernement irlandais, Institut pour l'environnement (University College, Dublin) et la Banque mondiale (EDIAR et AFTEN).

[78] Serpantié, G., Méral, P. and Bidaud, C. (2012) Des bienfaits de la nature aux services écosystémiques.

[79] Milošević, D.D., Savić, S.M., Marković, V., Arsenović, D. and Šećerov, I. (2016) Outdoor Human Thermal Comfort in Local Climate Zones of Novi Sad (Serbia) during Heat Wave Period. Hungarian Geographical Bulletin, 6, 129-137. https://doi.org/10.15201/hungeobull.65.2.4

[80] Molnár, V.É., Tóthmérész, B., Szabó, S. and Simon, E. (2018) Urban Tree Leaves' Chlorophyll-A Content as a Proxy of Urbanization. Air Quality, Atmosphere and Health, 11, 665-671. https://doi.org/10.1007/s11869-018-0573-5

[81] Ngom, D., Charahabil, M.M., Sarr, O., Bakhoum, A. and Akpo, L.E. (2014) Perceptions communautaires sur les services écosystémiques d'approvisionnement fournis par le peuplement ligneux de la Réserve de Biosphère du Ferlo (Sénégal).

[82] FAO (2001) Urban and Peri-Urban Forestry: Case Studies on Developing Countries. FAO, Rome.

[83] FAO (2012) Synthesis of Thematic Studies on Urban and Peri-Urban Forestry in N'Djaména, Chad: Support for the Formulation of an Urban and Peri-Urban Forestry Strategy and Action Plan in N'Djaména, Republic of Chad. Discussion Paper on Urban and peri-Urban Forestry $n^{\circ} 7$. FAO, Rome.

[84] Sjöstedt, M. (2012) Ecosystem Services and Poverty Reduction: How Do Development Practitioners Conceptualize the Linkages? The European Journal of Development Research, Palgrave Macmillan; European Association of Development Research and Training Institutes (EADI), 24, 777-787.

https://doi.org/10.1057/ejdr.2012.16

[85] Ouédraogo, I., Nacoulma, B.M.I., Hahn, K. and Thiombiano, A. (2014) Assessing Ecosystem Services Based on Indigenous Knowledge in South-Eastern Burkina Faso (West Africa). International Journal of Biodiversity Science, Ecosystem Services and Management, 10, 313-321. https://doi.org/10.1080/21513732.2014.950980

[86] Diop, M., Sambou, B., Goudiaby, A., Guiro, I. and Niang-Diop, F. (2011) Ressources végétales et préférences sociales en milieu rural sénégalais. Bois et Forêts des Tropiques, 310, 57-68. https://doi.org/10.19182/bft2011.310.a20459

[87] Walker, B.H., Carpentier, S., Anderies, A., Abel, N., Cumming, C., Janssen, M., Lebel, L., Norberg, J., Peterson, G.D. and Pritchard, R. (2002) Resilience Management in Social-Ecological Systems: A Working Hypothesis for a Participatory Approach. 
Conservation Ecology, 6, 1-14.

http://www.consecol.org/vol6/iss1/art14

https://doi.org/10.5751/ES-00356-060114

[88] Walker, B.H., Holling, C.S., Carpenter, S.R. and Kinzig, A.P. (2004) Resilience, Adaptability, and Transformability in Social-Ecological Systems. Ecology and Society, 9, 5. http://www.ecologyandsociety.org/vol9/iss2/art5/ https://doi.org/10.5751/ES-00650-090205

[89] Chevassus-au-Louis, B. and Pirard, R. (2011) Les services écosystémiques des forêts et leur rémunération éventuelle. Revue Forestière Française, 63, 579-599. https://doi.org/10.4267/2042/46106

[90] Chevassus-au-Louis, B., Salles, J.-M. and Pujol, J.L. (2009) Approche économique de la biodiversité et des services liés aux écosystèmes. Contribution à la décision publique. Rapport du groupe de travail présidé par Bernard Chevassus-au-Louis. Paris: Centre d'analyse stratégique; La Documentation française, 2009 (Études et Documents, $\left.\mathrm{n}^{\circ} 18\right)$

[91] Mishra, A. (2009) La lutte contre la pauvreté commence avec les ressources naturelles. In: Pierre Jacquet, et al., Eds., Regards sur la Terre, Presses de Sciences Po, Paris, 220-221.

https://www.cairn.info/regards-sur-la-terre-2009---page-220.htm

[92] Fisher, J.A., Patenaude, G., Giri, K., Lewis, K., Meir, P., Pinho, P., Rounsevell, M.D.A. and Williams, M. (2013) Understanding the Relationships between Ecosystem Services and Poverty Alleviation: A Conceptual Framework. Ecosystem Services, 7, 34-35. https://doi.org/10.1016/j.ecoser.2013.08.002

[93] Ingram, V., Awono, A. and Schure, J. (2016) Les PFNL participent à la création de revenus pour les ménages pauvres. In: Vivre et se nourrir de la forêt en Afrique centrale, FAO. Produits Forestiers Non-Ligneux, 21, 47-53.

[94] Tabuna, H. (2016) Immigration et développement des exportations des PFNL alimentaires d'Afrique centrale. In: Vivre et se nourrir de la forêt en Afrique centrale FAO. Produits Forestiers Non-Ligneux, 21, 57-63.

[95] Carpenter, S.R., Mooney, H.A., Agard, J., Capistrano, D., Defries, R.S., Diaz, S., Dietz, T., Duraiappah, A.K., Oteng-Yeboah, A., Pereira, H.M., Perrings, C., Reid, W.V., Sarukhan, J., Scholes, R.J. and Whyte, A. (2009) Science for Managing Ecosystem Services: Beyond the Millennium Ecosystem Assessment. Proceedings of the National Academy of Sciences of the United States of America, 106, 1305-1312. https://doi.org/10.1073/pnas.0808772106

[96] Rounsevell, M., Dawson, T. and Harrison, P. (2010) A Conceptual Framework to Assess the Effects of Environmental Change on Ecosystem Services. Biodiversity and Conservation, 19, 2823-2842. https://doi.org/10.1007/s10531-010-9838-5

[97] Shackleton, S., Chinyimba, A., Hebinck, P., Shackleton C. and Kaoma, H. (2015) Multiple Benefits and Values of Trees in Urban Landscapes in Two Towns in Northern South Africa. Landscape and Urban Planning, 136, 76-86.

https://doi.org/10.1016/j.landurbplan.2014.12.004

[98] Kimpouni, V., Nzila, J.D.D., Watha-Ndoudy, N., Kokolo Bilongo, E.C., Yallo Mouhamed, S., Kampe, J.-P. and Louembe, D. (2020) Sociocultural and Ecological Dynamics of Green Spaces in Brazzaville (Congo). International Journal of Ecology, 2020, 1-12. https://doi.org/10.1155/2020/3719267

[99] Savić, S., Unger, J., Gál, T., Milošević, D. and Popov, Z. (2013) Urban Heat Island Research of Novi Sad (Serbia). Geographica Pannonica, 17, 32-36.

https://doi.org/10.5937/GeoPan1301032S 
[100] Laille, P., Provendier, D. and Colson, F. (2013) Les bienfaits du végétal en villeSynthèse des travaux scientifiques et méthode d'analyse. Plante \& Cité: Ingénierie de la nature en ville, Plante et cité, Suisse.

[101] WHO (2004) Urbanisme et Santé. S2D/Association Internationale Pour la Promotion de la Santé et du Développement Durable. World Health Organisation, Geneva.

[102] Limoges, B. (2011) Les services rendus par la biodiversité. Forum Environnement de la FIHOQ, 10 février 2011, Ministère du Développement durable, de l'Environnement et des Parcs du Québec, Quebec. 